\title{
RANK-ONE QUANTUM GAMES
}

\author{
T. COONEY, M. JUNGE, C. PALAZUELOS, AND D. PÉREZ- GARCÍA
}

\begin{abstract}
In this work we study rank-one quantum games. In particular, we focus on the study of the computability of the entangled value $\omega^{*}$ and the entangled value with one-way quantum communication $\omega_{\text {qow }}$ of these games. We will show that the value $\omega^{*}$ can be efficiently approximated up to a 4-multiplicative factor and that $\omega_{\text {qow }}$ can be efficiently computed. We also study the behavior of these values under the parallel repetition of rank-one quantum games. We will show that $\omega^{*}$ does not verify a perfect parallel repetition theorem and that $\omega_{\text {qow }}$ does verify such a theorem.
\end{abstract}

\section{INTRODUCTION}

The study of two-prover one-round games is a central topic in both theoretical computer science and quantum information theory (QIT). Their importance in the first subject is mainly due to the fact that some of the most relevant problems in complexity theory can be stated in terms of these games. From the quantum information point of view, twoprover one-round games are a natural setting in which to understand Bell inequalities. Bell inequalities have always played a fundamental role in QIT and its applications cover a huge variety of topics.

A two-prover one-round game $G$ is specified by a referee, who chooses a pair of questions according to a probability distribution and who sends one question to each of the provers. These provers respond with answers taken from a certain finite set. The referee will decide whether the provers win according to a predicate which depends on the questions and answers. The provers can agree in advance on a strategy for their answers but they are not allowed to communicate with each other once the game has started. Computer scientists are mainly interested in the classical value of the game, $\omega(G)$, which is defined as the maximum attainable winning probability of the provers when they are allowed to use classical strategies. However, having in mind that quantum mechanics provides us with, in principle, new possibilities, one can consider the maximum attainable winning probability of the provers when they are allowed to share (unlimited) entanglement to define their strategies. We then talk of the entangled value of the game $G$ and we denote it by $\omega^{*}(G)$. One of Bell's fundamental observations can be reformulated as saying that $w^{*}(G) \geq \sqrt{2} w(G)>w(G)$ for certain games $G$ (so the provers can indeed define strictly better strategies if they are allowed to use quantum resources instead of just classical

The first, third and fourth authors are partially supported by the Spanish grants QUITEMAD, I-MATH, MTM2011-26912, S2009/ESP-1594 and the European project QUEVADIS. The second author is partially supported by NSF DMS-0901457. 
strategies). Moreover, the value $\omega^{*}(G)$ and the quotient $\frac{\omega^{*}(G)}{\omega(G)}$ have been shown to be very important parameters in QIT. This has motivated that some of the fundamental questions about $\omega(G)$ in computer science have been studied for the entangled value of the game $\omega^{*}(G)$. In particular, the study of how hard it is to compute or approximate the value $\omega^{*}(G)$ and the behavior of the parameter $\omega^{*}(G)$ with respect to the parallel repetition of $G$ has captured the attention of many authors in the few last years ([22], 6], [23], [24], [25]).

It turns out that another class of games naturally arises in the context of quantum information. Indeed, quantum games are those in which the communication between the referee and the provers (the questions and answers) is transmitted using quantum states. Specifically, in a quantum game the referee prepares an initial tripartite state $A B R$ and sends registers $A$ and $B$ to Alice and Bob (the provers), respectively. After following their previously agreed strategy, Alice and Bob will send back their new registers to the referee, who will test the answers via a two outputs (win/lose) projective measurement system. We will give a more precise explanation of these games in Section 3. In the same way as above, one can define different values for quantum games according to the strategies that Alice and Bob are allowed to use. Since everything considered in these games is quantum, the classical value of the game $\omega(G)$ does not seem so natural. However, we can define the entangled valued of a quantum game, $\omega^{*}(G)$, in exactly the same way as before. That is, $\omega^{*}(G)$ will be maximum attainable winning probability of the provers when they are allowed to share unlimited entanglement to define their strategies. Furthermore, we can also consider the value of the game when Alice and Bob are allowed to share unlimited entanglement and to send unlimited amount of quantum information. In this work we will restrict this to one-way communication. This means that we will allow one of the provers to send information to the other one, but not the other way around. We will talk in this case about the entangled value of the game with one-way communication and we will denote it by $\omega_{\text {qow }}(G)$. As we will show (see Subsection 3.2) the value $\omega_{\text {qow }}(G)$ can be much larger that $\omega(G)$ for certain rank-one quantum games $G$, so they indeed define different situations. Furthermore, it will be very interesting to define the maximal value of the game, $V(G)$, as the maximum attainable winning probability for a unique player with access to both Hilbert spaces (or, equivalently, when Alice and Bob both have all the information). It is very easy to see that the entangled value of the game with two way communication, so the case when both players can send an unlimited amount of quantum information, already matches the value $V(G)$. A much more surprising result was given by Buhrman et all ([4, Theorem 4.1]), who proved that in order to obtain the value $V(G)$ it is enough to consider the entangled value of the game with simultaneous mutual communication. That is, when Alice and Bob can both send an unlimited amount of quantum information but their message cannot depend on the ones received. Therefore, one could ask whether our second model, the entangled value with one-way communication, already 
matches the value $V(G)$. We will show in Subsection 3.2 that $V(G)$ is much larger than $\omega_{\text {qow }}(G)$ for some rank-one quantum games $G$.

Two recent papers have studied quantum games from different perspectives ([22], [28]). In the first work, the authors studied general quantum games. Following the approach mentioned before, they studied some of the important parameters in the context of computer science for the entangled value of quantum games. In particular, in [22] the authors studied the hardness of computing the value $\omega^{*}(G)$. One of the main results presented in that work states that it is NP-hard to approximate the entangled value of a general quantum game with inverse polynomial precision. On the other hand, the approach followed in [28] was via studying some particular quantum games. Indeed, motivated by the study of how much entanglement is needed to play optimally a quantum game, in [28] the authors restricted to a particular case of quantum games, the so called coherent state exchange games. Then, the authors showed that some of these quantum games can be played optimally with an infinite amount of entanglement (that is, $\omega^{*}(G)=1$ ), though no finite dimensional entangled state can lead to the same value of the game. It is very interesting to mention that there is not any known analogous result for the entangled value of classical games. The reader can find some other references on quantum games considering some other problems: A single prover ([37], [26]), limited prior entanglement ([27]) or provers dealing with classical communication but nor prior shared entanglement ([3]).

In this paper we will deal with those quantum games in which the projective measurement of the referee is defined by a rank-one projection. We will call these games rank-one quantum games. We note that a similar framework was studied in [34, which includes a formula (different from those considered in this paper) for the entangled value $\omega^{*}(G)$ of a rank one quantum game. One example of these games are the coherent state exchange games studied in [28]. Actually, we will introduce some other examples of rank-one quantum games presenting some interesting properties. Our approach to the study of these games is via operator spaces. Operator space theory can be understood as a non-commutative version of the classical Banach space theory. Following the philosophy that this expresses mathematically the description of quantum mechanics as a non-commutative version of classical theory, operator spaces have been shown to be a natural mathematical tool in quantum information theory. In recent years, they have been applied in several contexts like Bell inequalities ([29], [20], 18], [19]), quantum channels ([7], [15]) or entanglement theory ([16]). We also refer to [32] for a very nice survey on the topic. The main result of this work says that given a rank-one quantum game $G$, the entangled value $\omega^{*}(G)$ and the entangled value with one-way communication $\omega_{\text {qow }}(G)$ can be expressed, respectively, by means of the minimal and the Haagerup tensor norm in a certain tensor product operator spact]. With this connection at hand, we will be able to

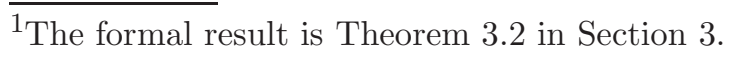


study both problems: the hardness of computing or approximating the values $\omega^{*}(G)$ and $\omega_{\text {qow }}(G)$, and the behavior of these two values with respect to the parallel repetition of the game. It will turn out that using some deep results already known in operator space theory, one can obtain the following Theorem 1.1 and Theorem 1.2 .

1.1. Computing and approximating the entangled value. Since the most important parameters in the study of games are the corresponding values (classical, entangled, oneway, ...), a lot of effort has been spent in order to know how hard these values are to compute or approximate. In the setting of classical games the problem is quite well understood if we focus on its classical value. Indeed, as a consequence of the PCP theorem ([1], 2] ) and the parallel repetition theorem of Raz [35], one can deduce that, unless $\mathrm{P}=\mathrm{NP}$, for any fixed $\epsilon>0$ there is no algorithm working in polynomial time in the number of questions and answers which can decide whether the value of a two-prover one-round game is 1 or $<\epsilon$.

Surprisingly, up to some results on particular kinds of games, nothing is known about the computability or approximability of the entangled value of a general two-prover oneround classical game2. The particular cases dealt with include XOR games, whose entangled value can be efficiently computed ([6]) and with unique games (a more general class than XOR games), whose entangled value can be efficiently approximated ([24]). Regarding quantum games, however, it was proved in [22] that it is NP-hard to approximate the entangled value $\omega^{*}(G)$ with inverse polynomial precision. In this paper we will study the computability of $\omega^{*}(G)$ and $\omega_{\text {qow }}(G)$ for rank-one quantum games $G$. We will show

\section{Theorem 1.1.}

1. The entangled value with quantum one-way communication $\omega_{\text {qow }}$ can be efficiently computed on rank-one quantum games.

2. The entangled value $\omega^{*}$ can be efficiently approximated up to a multiplicative constant relative error on rank-one quantum games.

Notice that, in contrast to the main result in [22] (which proves an NP-hardness result on $\omega^{*}(G)$ for general quantum games), Theorem 1.1 shows how to approximate the value $\omega^{*}(G)$ for rank-one quantum games. As we will show, the approximability result on $\omega^{*}(G)$ is based on a deep theorem in operator space theory which is a non-commutative version of Grothendieck's theorem. As far as we know, Theorem 1.1 is the first result giving a positive result in this direction. Since the proof in [22] does not apply to rank-one quantum games, we do not know if $\omega^{*}(G)$ can be efficiently computed or even approximated to polynomial precision.

1.2. Parallel repetition of the game. One of the most important problems in the study of classical games is, given a game $G$, how to decrease its value. This can be easily done if

${ }^{2}$ We must mention that in 22 the authors proved that it is NP-hard to approximate the entangled value of a three-prover one-round classical game, $\omega^{*}(G)$, with inverse polynomial precision. 
one allows to increase the number of rounds and/or the number of provers (just repeating the game sequentially and/or repeating the game in parallel with independent pairs of provers). However, the problem becomes much more difficult if we want to decrease the value of the game by using the same number of rounds and provers. The natural way to do this is to repeat the game many times in parallel. That is, in the setting of classical games the referee will choose $n$ pairs of questions independently and will send to each prover the corresponding $n$-tuple of questions. Then, each prover will respond a $n$-tuple of answers, which are accepted if each of the $n$ answer pairs would have been accepted in the original game. If we denote by $G^{n}$ the game played $n$ times in parallel, it is trivial to see that

$$
\omega\left(G^{n}\right) \geq \omega(G)^{n}
$$

Somewhat surprisingly, we do not have equality in (1.1) in general (see [10]). The problem of parallel repetition is then to find good upper bounds for the value $\omega\left(G^{n}\right)$. A long series of works on this problem culminated with the work of Raz ([35]), where he proved the so called parallel repetition theorem. That is, the value of a game repeated in parallel decreases exponentially with the number of repetitions $n$ (although not exactly at rate $\left.\omega(G)^{n}\right)$. We say that certain games verify a perfect parallel repetition theorem if $\omega\left(G^{n}\right)=$ $\omega(G)^{n}$ for every $n$. Note that this problem can also be stated exactly in the same way for the entangled value of a classical game $\omega^{*}(G)$. However, in this context the situation is not so well understood. In [6] the authors showed a perfect parallel repetition for the entangled value of XOR games. After that, in [24] the authors proved a parallel repetition theorem for unique games. Regarding the general situation, the best known result was given in the very recent work [25], where the authors showed that the value $\omega^{*}(G)$ can be indeed reduced through parallel repetition, provided it was not initially 1 . The best rate of decrease for the value $\omega^{*}(G)$ obtained by repeating the game is still an open problem.

In this work we will study the parallel repetition of a rank-one quantum game. Given a rank-one quantum game $G$, one can analogously define a parallel repetition of this game $G^{n}$ just by considering the tensor product of both the preparation state and the rank-one projection which defines the referee's test (in particular, a parallel repetition of a rank-one quantum game is again a rank-one quantum game). We will study here whether there exists a perfect parallel repetition for the values $\omega^{*}(G)$ and $\omega_{\text {qow }}(G)$ on rank- one quantum games. We will show

\section{Theorem 1.2.}

1. The entangled value with one-way communication verifies a perfect parallel repetition theorem on rank-one quantum games $G$ :

$$
\omega_{\text {qow }}\left(G^{n}\right)=\omega_{\text {qow }}(G)^{n}
$$


2. The entangled value does not verify a perfect parallel repetition theorem on rankone quantum games. Specifically, for every natural number $n$ there exists a rankone quantum game $G$ of local dimension $n$ for which

$$
\frac{\omega^{*}\left(G^{2}\right)}{\omega^{*}(G)^{2}} \succeq n^{2}
$$

where $\succeq$ denotes inequality up to a universal constant independent of $n$.

The first part of the previous theorem presents an optimal result. On the other hand, having in mind that we have a perfect parallel repetition theorem for the entangled value of XOR classical games, the second result is somehow surprising. It says that even in the most basic scenario of quantum games, the rank-one quantum games, we do not have a perfect parallel repetition theorem. Motivated by this fact, we will present a quite large family of rank-one quantum games for which perfect parallel repetition theorem is not far from being true. However, we will show that even for those games perfect parallel repetition fails.

To conclude, we mention that the techniques used in this paper also apply to the general case of quantum games. In particular, one can describe general quantum games via certain tensor norms in the framework of operator spaces. However, since the results for the general case require even more of the technology of tensor products, we defer the treatment of general quantum games to a forthcoming paper.

The paper is organized as follows. Section 2 is devoted to introducing the basic tools that we will need about operator spaces. In the first part of Section 3 we will explain in detail quantum games and the values $\omega^{*}, \omega_{\text {qow }}$; we will show the connections between these values and certain tensor norms in the category of operator spaces. In the second part of this section, we will explain some particularly interesting examples of rank-one quantum games. Sections 4 and 5 will be devoted, respectively, to proving Theorem 1.1 and Theorem 1.2. We will finally discuss our results and some open problems in Section 6.

\section{Operator Spaces}

We now recall some basic ideas from operator space theory; further details can be found in [9] and 31]. An operator space $V$ is a closed subspace of $B(H)$, the bounded operators on a Hilbert space $H$. The inclusions $M_{n}(V) \subseteq M_{n}(B(H)) \simeq B\left(H^{\otimes n}\right)$ induce matrix norms $\|\cdot\|_{n}$ on $M_{n}(V)$. By Ruan's Theorem (Theorem 2.3.5 in [9]), these matrix norms also characterize operator spaces. The existence of such an inclusion into $B(H)$ is equivalent to having a sequence of matrix norms $\left(M_{n}(V),\|\cdot\|_{n}\right)$ satisfying the following conditions:

- $\|v \oplus w\|_{m+n}=\max \left\{\|v\|_{m},\|w\|_{n}\right\}$ and

- $\|\alpha v \beta\|_{n} \leq\|\alpha\|\|v\|_{m}\|\beta\|$ 
for all $v \in M_{m}(V), w \in M_{n}(V), \alpha \in M_{n, m}$, and $\beta \in M_{m, n}$. To specify an operator space structure, one can either provide an explicit inclusion of $V$ into $B(H)$ or one can describe the matrix norms on $M_{n}(V)$.

A simple, but important, example of an operator space is $M_{N}$ with its operator space structure given by the usual identification $M_{N} \simeq B\left(\mathbb{C}^{N}\right)$. The matrix norm $\|\cdot\|_{n}$ on $M_{n}\left(M_{N}\right)$ is then the usual operator norm on $M_{n N}$.

Given operator spaces $V$ and $W$ and a linear map $T: V \rightarrow W$, let $T_{n}: M_{n}(V) \rightarrow$ $M_{n}(W)$ denote the linear map defined by

$$
T_{n}(v)=\left(i d_{n} \otimes T\right)(v)=\left(T\left(v_{i j}\right)\right) .
$$

A map is said to be completely bounded if

$$
\|T\|_{c b}=\sup _{n}\left\|T_{n}\right\|<\infty,
$$

and this quantity is then called the completely bounded norm of $T$. The map $T$ is said to be a complete isometry if each map $T_{n}$ is an isometry. In general, a Banach space will have different operator space structures that are not completely isometrically isomorphic to each other and it is necessary to specify which operator space structure is being used. We now describe the operator space structures which we will need in this article.

The set of completely bounded maps from $V$ to $W$ is denoted by $C B(V, W)$ and is again an operator space with matrix norms given by the identification

$$
M_{n}(C B(V, W))=C B\left(V, M_{n}(W)\right) .
$$

This allows us to define the dual operator space $V^{*}$ with matrix norms given by

$$
M_{n}\left(V^{*}\right)=M_{n}(C B(V, \mathbb{C}))=C B\left(V, M_{n}\right) .
$$

In particular, this defines an operator space structure on $S_{1}^{n}=M_{n}^{*}$. Note that this operator space structure is not given by the linear map identifying matrices in $S_{1}^{n}$ with matrices in $B\left(\mathbb{C}^{n}\right)$ as this map does not induce the correct norm on $S_{1}^{n}$. By Theorem 3.2 .3 in [9], the scalar pairing

$$
\langle b, c\rangle=\sum_{s, t} b_{s, t} c_{s, t}=\operatorname{Tr}\left(b c^{t r}\right)
$$

yields completely isometric isomorphisms $M_{n}^{*} \simeq S_{1}^{n}$ and $S_{1}^{n *} \simeq M_{n}$.

We now describe some of the different operator space structures that can be placed on the space $\ell_{2}^{n}$ : the column and row hilbertian operator spaces

$$
\begin{array}{ll}
C_{n}=\operatorname{span}\left\{e_{i 1} \mid 1 \leq i \leq n\right\} \subseteq M_{n}, & C=\overline{\operatorname{span}}\left\{e_{i 1} \mid i \in \mathbb{N}\right\} \subseteq B\left(\ell_{2}\right), \\
R_{n}=\operatorname{span}\left\{e_{1 i} \mid 1 \leq i \leq n\right\} \subseteq M_{n}, & R=\overline{\operatorname{span}}\left\{e_{1 i} \mid i \in \mathbb{N}\right\} \subseteq B\left(\ell_{2}\right)
\end{array}
$$


and $\mathrm{OH}_{n}$, the Operator Hilbert space with matrix norms given by

$$
\left\|\sum_{i=1}^{n} x_{i} \otimes e_{i}\right\|_{O H, N}=\left\|\sum_{i=1}^{n} x_{i} \otimes \bar{x}_{i}\right\|_{M_{N^{2}}}^{1 / 2},
$$

where $\sum_{i=1}^{n} x_{i} \otimes e_{i} \in M_{N}\left(\ell_{2}^{n}\right)$.

These Hilbertian operator space structures all agree at the Banach space level but differ as operator space structures; the norms $\|\cdot\|_{C},\|\cdot\|_{R},\|\cdot\|_{O H}$ are all equal to $\|\cdot\|_{\ell_{2}^{n}}$, but the matrix norms $\left(\|\cdot\|_{C, N},\|\cdot\|_{R, N},\|\cdot\|_{O H, N}\right)$ on $M_{N}\left(\ell_{2}^{n}\right)$ are different.

Given an embedding $V \subseteq B(H), \bar{V}$ is the same vector space but with the conjugate multiplication by a complex scalar and its conjugate operator space structure is given by the corresponding embedding $\bar{V} \subseteq \overline{B(H)}=B(\bar{H})$. Also, given a linear map $T: V \rightarrow W$ between operator spaces we can define $\bar{T}: \bar{V} \rightarrow \bar{W}$ as $\bar{T}(\bar{x})=\overline{T(x)}$ for every $\bar{x} \in \bar{V}$.

Taking the duals of the above operator spaces yields the completely isometric identifications

$$
C_{n}^{*}=R_{n}, \quad R_{n}^{*}=C_{n}, \quad O H_{n}^{*}=\overline{O H_{n}} .
$$

When we deal with an infinite dimensional Hilbert space $H$ (in particular, $\ell_{2}$ ) we write $R(H), C(H)$, and $O H(H)(R, C$, and $O H)$ and all the above identifications remain true.

Given two operator spaces $V \subseteq B(H)$ and $W \subseteq B(K)$, their algebraic tensor product $V \otimes W$ is a subspace of $B(H \otimes K)$ and their minimal operator space tensor product $V \otimes_{\min } W$ is the closure of $V \otimes W$ in $B(H \otimes K)$. If $V$ and $W$ are finite dimensional, then we have the following completely isometric identification.

$$
V \otimes_{\min } W=C B\left(V^{*}, W\right) .
$$

This corresponds to the Banach space identity $E \otimes_{\varepsilon} F=B\left(E^{*}, F\right)$ for finite-dimensional Banach spaces. We also note that if $u=\sum_{i=1}^{l} v_{i} \otimes w_{i} \in V \otimes W$, then

$$
\|u\|_{\text {min }}=\sup \left\{\left\|\sum_{i=1}^{l} T\left(v_{i}\right) \otimes S\left(w_{i}\right)\right\|_{B\left(H_{V} \otimes H_{W}\right)}\right\},
$$

where the supremum is taken over all finite dimensional Hilbert spaces $H_{V}$ and $H_{W}$ and complete contractions $T: V \rightarrow B\left(H_{V}\right)$ and $S: W \rightarrow B\left(H_{W}\right)$.

Given an element $u \in M_{n}(V \otimes W)$, its projective operator space tensor product norm is

$$
\|u\|_{\wedge}=\inf \{\|\alpha\|\|v\|\|w\|\|\beta\|: u=\alpha(v \otimes w) \beta\}
$$

where the infimum is taken over all decompositions with $v \in M_{p}(V), w \in M_{q}(W), \alpha \in$ $M_{n, p q}$, and $\beta \in M_{p q, n}$. The operator space projective tensor product $V \widehat{\otimes} W$ is the closure of $V \otimes W$ with respect to this norm.

If $V$ and $W$ are finite dimensional, we have the following duality relations:

$$
(V \widehat{\otimes} W)^{*}=V^{*} \otimes_{\min } W^{*} \quad \text { and } \quad\left(V \otimes_{\min } W\right)^{*}=V^{*} \widehat{\otimes} W^{*} .
$$


Note that, in particular, we have $S_{1}\left(H_{A}\right) \widehat{\otimes} S_{1}\left(H_{B}\right)=S_{1}\left(H_{A} \otimes H_{B}\right)$ for all finite dimensional Hilbert spaces $H_{A}$ and $H_{B}$.

Suppose that $V$ and $W$ are operator spaces and that $u=\sum_{k=1}^{l} a_{k} \otimes v_{k} \otimes w_{k} \in M_{N} \otimes$ $V \otimes W$. The Haagerup tensor product norm of $u$ is

$$
\|u\|_{h}=\sup \left\{\left\|\sum a_{k} \otimes \sigma_{V}\left(v_{k}\right) \sigma_{W}\left(w_{k}\right)\right\|_{M_{N} \otimes_{\min } B(H)}\right\},
$$

where the supremum is taken over all Hilbert spaces $H$ and complete contractions $\sigma_{V}$ : $V \rightarrow B(H), \sigma_{W}: W \rightarrow B(H)$. The completion of $V \otimes W$ with respect to this norm is denoted by $V \otimes_{h} W$. The Haagerup tensor product norm (which is associative but not commutative) has several remarkable properties including the following completely isometric identities:

- $R_{n} \otimes_{h} C_{n}=S_{1}^{n}, C_{n} \otimes_{h} R_{n}=M_{n}$,

- $V \otimes_{\min } R=V \otimes_{h} R, R \widehat{\otimes} V=R \otimes_{h} V$,

- $R=R \otimes_{h} R$,

where $C$ and $R$ are the column and row hilbertian operator spaces introduced earlier, and $V$ is an arbitrary operator space. We note that the Haagerup tensor product is both injective and projective (see 31] for further details). We will also need the following identities: $R_{n} \widehat{\otimes} C_{n}=S_{1}^{n}$ and $C_{n} \otimes_{\min } R_{n}=M_{n}$.

If $P: M_{n} \rightarrow R_{n}$ is the projection onto the first row, then $P^{*}: C_{n} \rightarrow S_{1}^{n}$ provides a completely isometric embedding of $C_{n}$ into $S_{1}^{n}$ given by $P^{*}\left(e_{i}\right)=e_{1 i}$ (mapping $C_{n}$ to the first row in $S_{1}^{n}$ ). Similarly, $R_{n}$ can be identified with the first column inside $S_{1}^{n}$. Taking the adjoint of the inclusion $\iota: R_{n} \rightarrow M_{n}$ yields a completely contractive projection from $S_{1}^{n}$ onto $C_{n}$ (and similarly there is a projection from $S_{1}^{n}$ onto $R_{n}$ ). Using the injectivity of the minimal and Haagerup tensor products and the projectivity of the projective operator space tensor product, we can then obtain completely isometric inclusions:

- $M_{n}=R_{n} \otimes_{\min } C_{n} \subseteq S_{1}^{n} \otimes_{\min } S_{1}^{n}$,

- $M_{n}=C_{n} \otimes_{h} R_{n} \subseteq S_{1}^{n} \otimes_{h} S_{1}^{n}$,

- $S_{1}^{n}=R_{n} \otimes_{h} C_{n} \subseteq S_{1}^{n} \otimes_{h} S_{1}^{n}$,

- $S_{1}^{n}=R_{n} \widehat{\otimes} C_{n} \subseteq S_{1}^{n} \widehat{\otimes} S_{1}^{n}$.

A linear map $u: V \rightarrow W$ is said to factor through $R$ if there are completely bounded maps $v: R \rightarrow W$ and $w: V \rightarrow R$ such that $u=v w . \Gamma_{R}(V, W)$ will denote the set of all such maps endowed with the norm

$$
\gamma_{R}(u)=\inf \left\{\|v\|_{c b}\|w\|_{c b}: u=v w\right\} .
$$

This becomes an operator space when $M_{n}\left(\Gamma_{R}(V, W)\right)$ is given the norm on $\Gamma_{R}\left(R_{n} \otimes_{\text {min }}\right.$ $\left.V, R_{n} \otimes_{\min } W\right)$.

By Proposition 5.16 from [31] and the remark following it, we have 
Proposition 2.1. Let $V$ and $W$ be finite-dimensional operator spaces. The natural mapping $V^{*} \otimes W \rightarrow C B(V, W)$ is a completely isometric isomorphism from $V^{*} \otimes_{h} W$ onto $\Gamma_{R}(V, W)$.

It is well known $([\underline{31}])$ that

$$
\Gamma_{R}\left(i d: C_{n} \rightarrow C_{n}\right)=\Gamma_{C}\left(i d: R_{n} \rightarrow R_{n}\right)=n .
$$

In particular, $\left\|\sum_{i=1}^{n} e_{i} \otimes e_{i}\right\|_{R_{n} \otimes_{h} C_{n}}=n$, while $\left\|\sum_{i=1}^{n} e_{i} \otimes e_{i}\right\|_{C_{n} \otimes_{h} R_{n}}=1$.

In the category of operator spaces, there are two natural ways of amplifying a bilinear map $\varphi: V \times W \rightarrow \mathbb{C}$, each with a different norm associated to it. The first is the joint amplification:

$$
M_{p}(V) \times M_{q}(W) \rightarrow M_{p q}, \quad\left(x_{i j}, y_{s t}\right) \mapsto\left(\varphi\left(x_{i j}, y_{s t}\right)\right)_{i, j, s, t},
$$

which leads to the following definition. A bilinear form $\varphi: V \times W \rightarrow \mathbb{C}$ is said to be jointly completely bounded if its linearization $\tilde{\varphi}$ is an element in $(V \widehat{\otimes} W)^{*}$ and $\|\varphi\|_{c b}$ is defined to be $\|\tilde{\varphi}\|_{(V \widehat{\otimes} W)^{*}}$. This corresponds to the Banach space identity $\operatorname{Bil}(E \times F, \mathbb{C})=\left(E \otimes_{\pi} F\right)^{*}$.

The second amplification has a formula corresponding to matrix multiplication:

$$
M_{p, l}(V) \times M_{l, q}(W) \rightarrow M_{p, q}, \quad\left(x_{i j}, y_{s t}\right) \mapsto\left(\varphi\left(\sum_{j=1}^{l} x_{i j} \otimes y_{j t}\right)\right)_{i, t} .
$$

A bilinear form $\varphi: V \times W \rightarrow \mathbb{C}$ is said to be completely bounded if its linearization $\tilde{\varphi}$ is an element in $\left(V \otimes_{h} W\right)^{*}$ and $\|\varphi\|_{c b}$ is defined to be $\|\tilde{\varphi}\|_{\left(V \otimes_{h} W\right)^{*}}$.

\section{Connections}

3.1. Rank-one quantum games and connections to operator spaces. We will start by explaining in detail rank-one quantum games. Actually, as we mentioned in Section 1, we will be interested in two different scenarios. In the first one, the two players, Alice and Bob, are allowed to use an entangled quantum state to define their strategy. In this case, the game works as follows:

(1) The referee, Charlie, prepares an initial state $|\psi\rangle \in H_{A} \otimes H_{B} \otimes H_{C}$ and sends the registers $A$ and $B$ to Alice and Bob, respectively.

(2) Alice and Bob also share (an arbitrary amount of) entanglement in the form of a state $|\varphi\rangle \in H_{A^{\prime}} \otimes H_{B^{\prime}}$. All Hilbert spaces are assumed to be finite-dimensional.

(3) Alice and Bob apply quantum operations $T_{A A^{\prime}}$ and $T_{B B^{\prime}}$ to $H_{A} \otimes H_{A^{\prime}}\left(\simeq H_{A A^{\prime}}\right)$ and $H_{B} \otimes H_{B^{\prime}}\left(\simeq H_{B B^{\prime}}\right)$ respectively.

(4) Let $|\gamma\rangle$ be a state in $H_{A} \otimes H_{B} \otimes H_{C}$. The triple $(A, B, C)$ is measured with respect to the projective measurement system with $P_{1}=|\gamma\rangle\langle\gamma|$ and $P_{0}=1-P_{1}$. The outcome 1 indicates that Alice and Bob win while 0 means that they lose.

(5) The game $G=G(|\psi\rangle,|\gamma\rangle)$ is completely determined by the initial state $|\psi\rangle$ and the measurement state $|\gamma\rangle$. 
(6) The value of the game is the supremum over all states $|\varphi\rangle$ and quantum operations $T_{A A^{\prime}}$ and $T_{B B^{\prime}}$ of the probability that Alice and Bob win the game. This value will be denoted by $\omega^{*}(G)$ and it will be called entangled value of $G$.

Our second scenario is that in which Alice and Bob are allowed to transmit information in one direction. In principle they can share an entangled state too but this can be incorporated in the communication. In this case, as before, we assume that Alice, Bob, and Charlie share an initial state $|\psi\rangle \in H_{A} \otimes H_{B} \otimes H_{C}$. After Alice and Bob perform their quantum operations, Charlie measures their responses against the measurement state $|\gamma\rangle \in H_{A} \otimes H_{B} \otimes H_{C}$. However, in this situation, Alice is allowed to communicate with Bob; they communicate via an auxiliary system $A^{\prime}$, initialized in the state $|\varphi\rangle \in H_{A^{\prime}}$. Alice applies a quantum operation to $H_{A} \otimes H_{A^{\prime}}$; Bob then applies a quantum operation to $H_{B} \otimes H_{A^{\prime}}$. The value of the game is again the supremum over all states $|\varphi\rangle$ and quantum operations $T_{A A^{\prime}}$ and $T_{B A^{\prime}}$ of the probability that Alice and Bob win the game. This value will be denoted by $\omega_{\text {qow }}(G)$ and it will be called entangled value of $G$ with one-way communication.

The main result of this paper is that operator spaces are ideally suited to describing the value of such quantum games. By taking a partial trace over the referee's register, we obtain a matrix $M_{A B}=t r_{C}|\psi\rangle\langle\gamma|$ in $S_{1}\left(H_{A}\right) \otimes S_{1}\left(H_{B}\right)$. Remarkably, by using different operator space tensor product norms, we can characterize those matrices corresponding to quantum games and describe the value of the quantum game attending the different resources the players are allowed to use.

In order to pave the way for the main result, we will start by showing that $S_{1}\left(H_{A}\right) \otimes$ $S_{1}\left(H_{B}\right)$ is indeed the natural space in which to realize the rank-one quantum games.

Proposition 3.1. Let $x \in S_{1}\left(H_{A} \otimes H_{B}\right)$. Then $\|x\|_{1} \leq 1$ if and only if there exist a finite dimensional Hilbert space $H_{C}$ and $|\psi\rangle,|\gamma\rangle$ in the unit sphere of $H_{A} \otimes H_{B} \otimes H_{C}$ such that $x=\operatorname{tr}_{C}|\psi\rangle\langle\gamma|$.

Proof. It is very easy to see that $x=t r_{C}|\psi\rangle\langle\gamma|$ is in the unit ball of $S_{1}\left(H_{A} \otimes H_{B}\right)$ for every states $|\psi\rangle,|\gamma\rangle \in H_{A} \otimes H_{B} \otimes H_{C}$. To see the converse, let's consider the SVD of $x=\sum_{i=1}^{N} \alpha_{i}\left|f_{i}\right\rangle\left\langle g_{i}\right|$, where $\left(f_{i}\right)_{i=1}^{N}$ and $\left(g_{i}\right)_{i=1}^{N}$ are orthonormal bases of $H_{A} \otimes H_{B}$ and $\left(\alpha_{i}\right)_{i=1}^{N}$ is a sequence of positive real numbers verifying $\sum_{i=1}^{N} \alpha_{i} \leq 1$. Then, we can consider $H_{C}=\ell_{2}^{N}$ and define $|\psi\rangle=\sum_{i=1}^{N} \sqrt{\alpha_{i}}\left|f_{i}\right\rangle|i\rangle$ and $|\gamma\rangle=\sum_{i=1}^{N} \sqrt{\alpha_{i}}\left|g_{i}\right\rangle|i\rangle$, where $(|i\rangle)_{i=1}^{N}$ is the canonical basis of $\ell_{2}^{N}$. It is trivial that $|\psi\rangle$ and $|\gamma\rangle$ are in the unit ball of $H_{A} \otimes H_{B} \otimes H_{C}$ and $x=\operatorname{tr}_{C}|\psi\rangle\langle\gamma|$. Finally, note that we can assume that $|\psi\rangle$ and $|\gamma\rangle$ have both norm one just by considering $H_{C}=\ell_{2}^{N+2}$ to complete norms.

Proposition 3.1 says that there is a one-to one correspondence between the unit ball of $S_{1}\left(H_{A}\right) \widehat{\otimes} S_{1}\left(H_{B}\right)$ and the set of rank-one quantum games via the matrices $M_{A B}=$ $\operatorname{tr}_{C}|\psi\rangle\langle\gamma|$. 
For any game $G=G(|\psi\rangle,|\gamma\rangle\langle\gamma|), V(G)=\left\|M_{A B}\right\|_{S_{1}\left(H_{A}\right) \widehat{\otimes} S_{1}\left(H_{B}\right)}^{2}$ will be called the maximal value of the game, since it represents the success probability of the game for one player that has access to both Hilbert spaces $H_{A}$ and $H_{B}$ (so the best possible situation).

The main connection in this work states that the minimal tensor norm and the Haagerup tensor norm give us respectively the entangled value of the game and the entangled value of the game with one-way communication.

Theorem 3.2. Let $G$ be a quantum entangled game with initial state $|\psi\rangle$ and final measurement $|\gamma\rangle\langle\gamma|$ for $|\psi\rangle,|\gamma\rangle \in H_{A} \otimes H_{B} \otimes H_{C}$. Let $M_{A B}=t_{C}|\psi\rangle\langle\gamma|$. Then,

1. The entangled value of the game is given by

$$
\omega^{*}(G)=\left\|M_{A B}\right\|_{S_{1}^{A} \otimes_{m i n} S_{1}^{B}}^{2} .
$$

2. The entangled value of the game with one-way communication from Alice to Bob is given by

$$
\omega_{\text {qow }}(G)=\left\|M_{A B}\right\|_{S_{1}^{A} \otimes_{h} S_{1}^{B}}^{2} .
$$

3. The maximal value of the game is given by

$$
V(G)=\left\|M_{A B}\right\|_{S_{1}^{A} \widehat{\otimes} S_{1}^{B}} .
$$

We will use the following lemma.

Lemma 3.3. Given an element $M \in S_{1}\left(H_{A}\right) \otimes S_{1}\left(H_{B}\right)$, we have that

1. $\|M\|_{S_{1}\left(H_{A}\right) \otimes_{m i n} S_{1}\left(H_{B}\right)}=\sup \left\{\left|\operatorname{tr}\left(\left\langle\xi\left|U_{A A^{\prime}} \otimes V_{B B^{\prime}}\right| \eta\right\rangle M\right)\right|\right\}$, where the supremum is taken over $H_{A^{\prime}}$ and $H_{B^{\prime}}$ finite dimensional Hilbert spaces, $|\xi\rangle,|\eta\rangle \in H_{A^{\prime} B^{\prime}}$, and $U_{A A^{\prime}}, V_{B B^{\prime}}$ are unitaries on $H_{A A^{\prime}}$ and $H_{B B^{\prime}}$ respectively.

2. $\|M\|_{S_{1}\left(H_{A}\right) \otimes_{h} S_{1}\left(H_{B}\right)}=\sup \left\{\left|\operatorname{tr}\left(\left\langle\xi\left|V_{B A^{\prime}} U_{A A^{\prime}}\right| \eta\right\rangle M\right)\right|\right\}$, where the supremum is taken over $H_{A^{\prime}}$ finite dimensional Hilbert space, $|\xi\rangle,|\eta\rangle \in H_{A^{\prime}}$, and $U_{A A^{\prime}}, V_{B A^{\prime}}$ are unitaries on $H_{A A^{\prime}}$ and $H_{B A^{\prime}}$ respectively.

3. Given an element $M \in S_{1}\left(H_{A} \otimes H_{B}\right)$, we have that

$$
\|M\|_{S_{1}\left(H_{A} \otimes H_{B}\right)}=\sup \{|\operatorname{tr}(\langle\xi|W| \eta\rangle M)|\},
$$

where the supremum is taken over finite dimensional Hilbert spaces $H_{E}$, unit vectors $|\xi\rangle,|\eta\rangle \in H_{E}$ and $W$ a unitary on $H_{A} \otimes H_{B} \otimes H_{E}$.

We will postpone the proof of the previous lemma to Section 7 .

Proof of Theorem 3.2.

1. After Alice and Bob perform their quantum operations, we are left with the state:

$$
\left(T_{A A^{\prime}} \otimes T_{B B^{\prime}}\right)(|\psi\rangle|\varphi\rangle\langle\psi|\langle\varphi|),
$$

and the probability of winning is given by

$$
\operatorname{tr}\left(\left(P_{1} \otimes 1_{A^{\prime} B^{\prime}}\right)\left(T_{A A^{\prime}} \otimes T_{B B^{\prime}}\right)(|\psi\rangle|\varphi\rangle\langle\psi|\langle\varphi|)\right)
$$


It follows from Stinespring's Theorem that we can write

$$
T_{A A^{\prime}}(\rho)=\operatorname{tr}_{\mathcal{K}}\left(U_{A A^{\prime} \mathcal{K}}(\rho \otimes|0\rangle\langle 0|) U_{A A^{\prime} \mathcal{K}}^{\dagger}\right),
$$

for a finite-dimensional Hilbert space $\mathcal{K}$ and a unitary $U_{A A^{\prime} \mathcal{K}}$ on $H_{A} \otimes H_{A^{\prime}} \otimes \mathcal{K}$ (and similarly for $\left.T_{B B^{\prime}}\right)$. It thus suffices to consider quantum operations of the form $T(x)=U x U^{\dagger}$, where $U$ is a unitary (by changing $|\varphi\rangle$ to $|\varphi\rangle|00\rangle$ and increasing the dimensions of $H_{A^{\prime}}$ and $H_{B^{\prime}}$ ).

The value of the game is then given by

$$
\sup _{U_{A A^{\prime}}, V_{B B^{\prime}},|\varphi\rangle} \operatorname{tr}\left(\left(P_{1} \otimes 1_{A^{\prime} B^{\prime}}\right)\left(U_{A A^{\prime}} \otimes V_{B B^{\prime}} \otimes 1_{C}\right)|\psi\rangle|\varphi\rangle\langle\psi|\langle\varphi|\left(U_{A A^{\prime}}^{\dagger} \otimes V_{B B^{\prime}}^{\dagger} \otimes 1_{C}\right)\right),
$$

where $U_{A A^{\prime}}$ and $V_{B B^{\prime}}$ are unitaries on the indicated Hilbert spaces.

Recalling that $P_{1}=|\gamma\rangle\langle\gamma|$, we can rewrite the above as

$$
\sup _{\left.U_{A A^{\prime}}, V_{B B^{\prime}}, \varphi\right\rangle} \|\left(\langle\gamma| \otimes 1_{A^{\prime} B^{\prime}}\right)\left(U_{A A^{\prime}} \otimes V_{B B^{\prime}} \otimes 1_{C}\right)|\psi\rangle|\varphi\rangle \|_{H_{A^{\prime}} \otimes H_{B^{\prime}}}^{2} .
$$

Taking the supremum over $|\varphi\rangle \in H_{A^{\prime}} \otimes H_{B^{\prime}}$, we can write this as an operator norm:

$$
\begin{aligned}
& \sup _{U_{A A^{\prime}}, V_{B B^{\prime}}}\left\|\left(\langle\gamma| \otimes 1_{A^{\prime} B^{\prime}}\right)\left(U_{A A^{\prime}} \otimes V_{B B^{\prime}} \otimes 1_{C}\right)\left(|\psi\rangle \otimes 1_{A^{\prime} B^{\prime}}\right)\right\|_{B\left(H_{A^{\prime}} \otimes H_{B^{\prime}}\right)}^{2} \\
= & \sup _{U_{A A^{\prime}}, V_{B B^{\prime}}}\left\|\operatorname{tr} r_{A B}\left(\left(U_{A A^{\prime}} \otimes V_{B B^{\prime}}\right) \operatorname{tr} C(|\psi\rangle\langle\gamma|) \otimes 1_{A^{\prime} B^{\prime}}\right)\right\|_{B\left(H_{A^{\prime}} \otimes H_{B^{\prime}}\right)}^{2} .
\end{aligned}
$$

For the sake of brevity, we will write $M_{A B}=\operatorname{tr}_{C}(|\psi\rangle\langle\gamma|)$. We can then evaluate the norm of this operator in $B\left(H_{A^{\prime}} \otimes H_{B^{\prime}}\right)$ by taking the supremum over elements in the unit ball of $S_{1}\left(H_{A^{\prime}} \otimes H_{B^{\prime}}\right)$. It suffices to take the supremum over elements of the form $\omega_{\xi, \eta}(x)=\langle\xi|x| \eta\rangle$ with $|\xi\rangle,|\eta\rangle$ in the unit ball of $H_{A^{\prime} \otimes B^{\prime}}$. We note that applying this element commutes with the partial trace $\operatorname{tr}_{A B}$. We then have that

$$
\omega^{*}(G)^{1 / 2}=\sup _{U_{A A^{\prime}}, V_{B B^{\prime}},|\xi\rangle,|\eta\rangle}\left|\operatorname{tr}_{A B}\left(\left\langle\xi\left|U_{A A^{\prime}} \otimes V_{B B^{\prime}}\right| \eta\right\rangle M_{A B}\right)\right| \cdot
$$

Applying Lemma 3.3 we conclude that

$$
\omega^{*}(G)^{1 / 2}=\left\|M_{A B}\right\|_{S_{1}^{A} \otimes_{\min } S_{1}^{B} .}
$$

2. Reasoning similarly to above, we obtain that the value of the game is given by

$$
\sup _{U_{A A^{\prime}}, V_{B A^{\prime}},|\varphi\rangle} \|\left(\langle\gamma| \otimes 1_{A^{\prime}}\right)\left(V_{B A^{\prime}} U_{A A^{\prime}} \otimes 1_{C}\right)|\psi\rangle|\varphi\rangle \|_{H_{A^{\prime}}}^{2},
$$

where $U_{A A^{\prime}}$ and $V_{B A^{\prime}}$ are unitaries on the indicated Hilbert spaces. Rearranging as before and denoting again $M_{A B}=\operatorname{tr}_{C}(|\psi\rangle\langle\gamma|)$, we can write this as

$$
\sup _{U, V,|\xi\rangle,|\eta\rangle}\left|\operatorname{tr}_{A B}\left(M_{A B}\left\langle\xi\left|V_{B A^{\prime}} U_{A A^{\prime}}\right| \eta\right\rangle\right)\right|^{2}
$$

where $|\xi\rangle,|\eta\rangle \in H_{A^{\prime}}$. Applying Lemma 3.3 we conclude that

$$
\omega_{\text {qow }}(G)^{1 / 2}=\left\|M_{A B}\right\|_{S_{1}^{A} \otimes_{h} S_{1}^{B}} .
$$


3. Reasoning similarly to above, we replace the unitaries $U \in B\left(H_{A} \otimes H_{A^{\prime}}\right)$ and $V \in$ $B\left(H_{B} \otimes H_{B^{\prime}}\right)$ by a single unitary $W \in B\left(H_{A} \otimes H_{B} \otimes H_{E}\right)$, where $H_{E}$ is the Hilbert space corresponding to the entangled state available to the player. Rearranging as before, we have that

$$
V(G)=\sup _{W,|\xi\rangle,|\eta\rangle}\left|\operatorname{tr}_{A B}\left(M_{A B}\left\langle\xi\left|W_{A B E}\right| \eta\right\rangle\right)\right|^{2},
$$

where $|\xi\rangle,|\eta\rangle \in H_{E}$. Applying Lemma 3.3, we conclude that

$$
V(G)^{1 / 2}=\left\|M_{A B}\right\|_{S_{1}^{A} \widehat{\otimes} S_{1}^{B}}
$$

Note that we have $0 \leq \omega^{*}(G) \leq \omega_{\text {qow }}(G) \leq V(G) \leq 1$ for every game $G$. However, Alice and Bob cannot define, in general, a perfect strategy. Indeed, the maximal value of the game $V(G)$ is in general strictly smaller than 1 . Actually, one can easily characterize those rank-one quantum games with maximal value equal to 1 . We will postpone the proof of the following lemma to Section 7.

Lemma 3.4. Consider the states $|\psi\rangle=\sum_{i} \lambda_{i}|i\rangle_{C}\left|\alpha_{i}\right\rangle_{A B}$ and $|\gamma\rangle=\sum_{i} \mu_{i}|i\rangle_{C}\left|\beta_{i}\right\rangle_{A B}$. The matrix $M_{A B}=\operatorname{tr}_{C}|\psi\rangle\langle\gamma|$ satisfies $\left\|M_{A B}\right\|_{S_{1}\left(H_{A}\right) \widehat{\otimes} S_{1}\left(H_{B}\right)}=1$ if and only if there is a unitary $U$ on $H_{A} \otimes H_{B}$ such that $\left|\alpha_{i}\right\rangle=U\left|\beta_{i}\right\rangle$ and $\lambda_{i}=\mu_{i}$ for all $i$.

3.2. The three models are different. Another important question is to show that the three values $\omega^{*}, \omega_{\text {qow }}$ and $V$ are indeed different. We will show in the following that for some rank-one quantum games $G$ in $S_{1}^{n} \otimes S_{1}^{n}$ we have $\frac{V(G)}{\omega_{\text {qow }}(G)} \geq n^{2}$ and for some some rank-one quantum games $G$ in $S_{1}^{n} \otimes S_{1}^{n}$ we have $\frac{\omega_{\text {qow }}(G)}{\omega^{*}(G)} \geq n^{2}$. We must also point out that though we will invoke Theorem 3.2 to translate the problem to the language of tensor norms on $S_{1}^{n} \otimes S_{1}^{n}$, the elements that we will use to separate the norms (and thus the models) are extremely easy and the corresponding game $G$ can easily be found.

Let's start showing that the maximal and the one-way values of a rank-one quantum game $G$ can be actually very different. According to Theorem 3.2 this is immediately deduced from the fact that

$$
\left\|i d: S_{1}^{n} \otimes_{h} S_{1}^{n} \rightarrow S_{1}^{n} \widehat{\otimes} S_{1}^{n}\right\| \geq n .
$$

To see this, consider the element $G_{R}=\sum_{i=1}^{n} e_{1 i} \otimes e_{i 1} \in S_{1}^{n} \otimes S_{1}^{n}$. It is very easy to see that $\left\|G_{R}\right\|_{S_{1}^{n} \otimes_{h} S_{1}^{n}}=1$ while $\left\|G_{R}\right\|_{S_{1}^{n} \widehat{\otimes} S_{1}^{n}}=n$. Indeed, the first estimate follows from the fact that $\left\|G_{R}\right\|_{S_{1}^{n} \otimes_{h} S_{1}^{n}}=\left\|\sum_{i=1}^{n} e_{i} \otimes e_{i}\right\|_{C_{n} \otimes_{h} R_{n}}=\left\|\sum_{i=1}^{n} e_{i i}\right\|_{M_{n}}=1$. On the other hand, $\left\|G_{R}\right\|_{S_{1}^{n} \widehat{\otimes} S_{1}^{n}}=\left\|\sum_{i=1}^{n} e_{i} \otimes e_{i}\right\|_{C_{n} \widehat{\otimes} R_{n}}=\left\|\sum_{i=1}^{n} e_{i i}\right\|_{S_{1}^{n}}=n$.

We now compare the one-way and entangled values of a rank-one quantum game. According to Theorem 3.2 it suffices to show that

$$
\left\|i d: S_{1}^{n} \otimes_{\min } S_{1}^{n} \rightarrow S_{1}^{n} \otimes_{h} S_{1}^{n}\right\| \geq n .
$$


To see this, consider the element $G_{C}=\sum_{i=1}^{n} e_{i 1} \otimes e_{1 i} \in S_{1}^{n} \otimes S_{1}^{n}$. It can be seen that $\left\|G_{C}\right\|_{S_{1}^{n} \otimes_{\min } S_{1}^{n}}=\left\|\sum_{i=1}^{n} e_{i} \otimes e_{i}\right\|_{R_{n} \otimes_{\min } C_{n}}=\left\|\sum_{i=1}^{n} e_{i i}\right\|_{M_{n}}=1$ and $\left\|G_{C}\right\|_{S_{1}^{n} \otimes_{h} S_{1}^{n}}=$ $\left\|\sum_{i=1}^{n} e_{i} \otimes e_{i}\right\|_{R_{n} \otimes_{h} C_{n}}=n$ (see Equation 2.1).

Finally it is worth mentioning that the previous separations are optimal. That is, using that $d_{c b}\left(M_{n}, R_{n^{2}}\right)=d_{c b}\left(S_{1}^{n}, R_{n^{2}}\right)=n$ one can easily show that

$$
\left\|i d: S_{1}^{n} \otimes_{h} S_{1}^{n} \rightarrow S_{1}^{n} \widehat{\otimes} S_{1}^{n}\right\| \leq n \text { and }\left\|i d: S_{1}^{n} \otimes_{\min } S_{1}^{n} \rightarrow S_{1}^{n} \otimes_{h} S_{1}^{n}\right\| \leq n .
$$

3.3. Schur Multiplier Games. In this section we will consider an interesting example of rank-one quantum games, the Schur Multiplier games.

Definition 3.1. Let $G$ be a quantum entangled game with initial state $|\psi\rangle$ and final measurement $|\gamma\rangle\langle\gamma|$ for $|\psi\rangle,|\gamma\rangle \in H_{A} \otimes H_{B} \otimes H_{C}$. Let $M_{A B}=\operatorname{tr}_{C}|\psi\rangle\langle\gamma|$. We will say that $G$ is a $S$ chur game if the associated map $\hat{M}_{A B}: B\left(H_{A}\right) \rightarrow S_{1}\left(H_{B}\right)$ to $M_{A B}$ is a Schur multiplier. That is, there exist an element $\Lambda \in B\left(H_{A}\right)$ verifying that

$$
\hat{M}_{A B}(X)=\Lambda * X
$$

for every $X \in B\left(H_{A}\right)$. Here, * denotes the Schur (or Hadamard) product.

Remark 3.1. Note that the dimensions of Alice and Bob's Hilbert spaces are the same for Schur games.

We will start by characterizing the games which correspond to Schur multipliers.

Lemma 3.5. A rank-one quantum game is a Schur game if and only if it has a representation $M=\operatorname{tr}_{C}|\psi\rangle\langle\gamma|$ where $|\psi\rangle$ and $|\gamma\rangle$ are of the form

$$
\begin{aligned}
|\psi\rangle & =\sum_{i, t} \alpha_{i t}|i\rangle_{A}|i\rangle_{B}|t\rangle_{C}, \\
|\gamma\rangle & =\sum_{i, t} \beta_{i t}|i\rangle_{A}|i\rangle_{B}|t\rangle_{C},
\end{aligned}
$$

with $\|\alpha\|_{2} \leq 1,\|\beta\|_{2} \leq 1$.

We will postpone the proof of Lemma 3.5 to Section 7

Although the entangled and one-way values of rank-one quantum games are in general very different, Schur games provide a nontrivial class of games for which these values are equivalent, i.e.,

$$
\frac{1}{4} \omega_{\text {qow }}(G) \leq \omega^{*}(G) \leq \omega_{\text {qow }}(G) .
$$

The equivalence of the corresponding tensor norms for Schur multipliers follows from a Grothendieck inequality for operator spaces and is proved in Theorem 4.2 in [33]. The inequality above follows from the improved constants for this Grothendieck inequality that are provided by [14.

It is important to notice here that, even when the entangled value with no communication and with one-way communication are the same, the amount of resources needed 
to attain such values can be very different in both situations. An extremal example is given by the game of Leung, Toner, and Watrous [28]. In this case the initial state is $|\psi\rangle=\frac{1}{\sqrt{2}}|000\rangle+\frac{1}{2}|1\rangle(|11\rangle+|22\rangle)$ whereas the final state is $|\gamma\rangle=\frac{1}{\sqrt{2}}(|000\rangle+|111\rangle)$. By the previous lemma, this is a Schur multiplier game. They show in [28] that one needs infinite entanglement in order to play the optimal strategy for the entangled value $\omega^{*}(G)$ which is in this case equal to 1 . It is however straightforward to see that with an auxiliary system $A^{\prime}$ of dimension 2, if Alice applies to $A A^{\prime}$ a unitary with $|00\rangle \mapsto|00\rangle$, $|10\rangle \mapsto|11\rangle$, and $|20\rangle \mapsto|10\rangle$ and later Bob applies on $B A^{\prime}$ a unitary with $|00\rangle \mapsto|00\rangle$, and $\frac{1}{\sqrt{2}}(|11\rangle+|20\rangle) \mapsto|10\rangle$, they can obtain $|\gamma\rangle$ starting from $|\psi\rangle$ with the communication of just one qubit.

\section{Computing And APPROXimating NORMS}

This section is devoted to prove Theorem 1.2. First, we will say that given a rank-one quantum game $G$ its size $|G|$ is given by the dimension of the Hilbert space to define the questions and the answers. In our case, this is $n$. With this at hand, we say that certain parameter can be computed or approximated efficiently if we can do it in a time which is polynomial in $|G|$.

Theorem 1.2 follows from the following theorem.

Theorem 4.1. Let $G$ be a quantum entangled game with initial state $|\psi\rangle$ and final measurement $|\gamma\rangle\langle\gamma|$ for $|\psi\rangle,|\gamma\rangle \in \ell_{2}^{n} \otimes \ell_{2}^{n} \otimes H_{C}$. Let $M_{A B}=\operatorname{tr}_{C}|\psi\rangle\langle\gamma|$. Then,

1. $V(G)$ can be computed efficiently.

2. $\omega_{\text {qow }}(G)$ can be computed efficiently.

3. There exists a value $\bar{\omega}(G)$ which can be computed efficiently and such that

$$
\frac{1}{4} \bar{\omega}(G) \leq \omega^{*}(G) \leq \bar{\omega}(G)
$$

The fact that the maximal value of a game $V(G)$ can be computed efficiently is very easy. Just note that $V(G)=\left\|M_{A B}\right\|_{S_{1}\left(H_{A} \otimes H_{A}\right)}$, so it is reduced to perform a SVD decomposition in the matrix $M_{A B}$. Therefore, the interesting part of Theorem 4.1 are points 2. and 3. Actually, the hard work for the value $\omega_{\text {qow }}(G)$ was already done in Theorem 3.2. Once we know that this value can be expressed as the Haagerup tensor norm on $S_{1}\left(H_{A}\right) \otimes S_{1}\left(H_{B}\right)$, the result follows from the following result:

Theorem 4.2 ([36], [15]). The completely bounded norm of $T: M_{n} \rightarrow M_{n}$ can be computed efficiently. Moreover, such a norm can be expressed by a semidefinite program.

To see how this result connects to ours, first note that there is an isometric identification between $B\left(H_{A}\right) \otimes_{h} B\left(H_{B}\right)$ and $C B\left(B\left(H_{a}\right), B\left(H_{b}\right)\right)$. Indeed, the map

$$
\Delta: B\left(H_{A}\right) \otimes_{h} B\left(H_{B}\right) \rightarrow C B\left(B\left(H_{A}\right), B\left(H_{B}\right)\right),
$$

defined by $\Delta\left(\sum_{i} x_{i} \otimes y_{i}\right)(A)=\sum_{i} x_{i} A y_{i}$ is an isometric isomorphism. Therefore, Theorem 4.2 states that $B\left(H_{A}\right) \otimes_{h} B\left(H_{B}\right)$ can be computed efficiently. On the other hand, this 
implies that the dual norm, $S_{1}\left(H_{A}\right) \otimes_{h} S_{1}\left(H_{B}\right)$, can be also computed in polynomial time (see [11] for details).

The key result in showing how to approximate the entangled value up to constant precision is the following Grothendieck inequality for operator spaces.

Theorem 4.3 ([33], [14]). Given two finite dimensional Hilbert spaces $H_{a}, H_{b}$ and an element $M \in S_{1}\left(H_{A}\right) \otimes S_{1}\left(H_{B}\right)$ we have

$$
\frac{1}{2}\|M\|_{*} \leq\|M\|_{S_{1}\left(H_{A}\right) \otimes_{\min } S_{1}\left(H_{B}\right)} \leq\|M\|_{*},
$$

where we define $\|M\|_{*}=\inf \left\{\|u\|_{S_{1}\left(H_{A}\right) \otimes_{h} S_{1}\left(H_{B}\right)}+\left\|v^{t}\right\|_{S_{1}\left(H_{A}\right) \otimes_{h} S_{1}\left(H_{B}\right)}: M=u+v\right\}$. Here $v^{t}$ is the transpose of $v$.

This version of the theorem can be obtained from the "standard one" (see [14, Theorem 1.1]) and Lemma 3.1 in [14] (see [14, Remark 3.2]). The result was first proved by Pisier and Shlyakhtenko ([33]) with a worse universal constant and then improved by Haagerup and Musat $([14])$. Next, we show that $\|\cdot\|_{*}$ can be computed in polynomial time. To do this, we use the fact that

$$
\|M\|_{*}=\sup \left\{\langle M, A\rangle: \max \left\{\|A\|_{B\left(H_{A}\right) \otimes_{h} B\left(H_{B}\right)},\left\|A^{t}\right\|_{B\left(H_{A}\right) \otimes_{h} B B}\right\} \leq 1\right\} .
$$

On the other hand, we have already seen that computing $\|A\|_{B\left(H_{A}\right) \otimes_{h} B\left(H_{B}\right)}$ is a semidefinite program (so max $\left\{\|A\|_{B\left(H_{A}\right) \otimes_{h} B\left(H_{B}\right)},\left\|A^{t}\right\|_{B\left(H_{A}\right) \otimes_{h} B\left(H_{B}\right)}\right\}$ is). Thus, it can be computed in polynomial time. As we have mentioned before, this property is preserved under polarity (see [1] ), so $\|\cdot\|_{*}$ can be computed in polynomial time.

\section{Parallel Repetition of Rank-One quantum games}

In this section we will prove Theorem 1.2. First, let's assume that we have a rank- one quantum game $G$ with initial state $|\psi\rangle$ and final measurement $P=|\gamma\rangle\langle\gamma|$ for $|\psi\rangle,|\gamma\rangle \in$ $H_{A} \otimes H_{B} \otimes H_{C}$. Let $M_{A B}=t r_{C}|\psi\rangle\langle\gamma| \in S_{1}^{n} \otimes S_{1}^{n}$. Then, the parallel repetition of this game $k$ times is given by the rank-one quantum game $G^{k}$ defined by an initial state $|\psi\rangle^{k}$ and final measurement $P^{\otimes_{k}}$. It is easy to see that this corresponds to the element $M_{A B}^{k}=\operatorname{tr}_{C}|\psi\rangle^{k}\left\langle\left.\gamma\right|^{k} \in S_{1}^{n^{k}} \otimes S_{1}^{n^{k}}\right.$ obtained by the $k^{t h}$ tensor product of $M_{A B}$. Theorem 1.2 follows trivially from the following result.

\section{Theorem 5.1.}

1. The maximal value $V$ verifies a perfect parallel repetition theorem on rank-one quantum games.

2. The entangled value with one-way communication $\omega_{\text {qow }}$ verifies a perfect parallel repetition theorem on rank-one quantum games. 
3. The entangled value does not verify a perfect parallel repetition theorem on rankone quantum games. Furthermore, for every natural number $n$ there exists a rankone quantum game $G$ of local dimension $n$ for which

$$
\frac{\omega^{*}\left(G^{2}\right)}{\omega^{*}(G)^{2}} \succeq n^{2}
$$

where $\succeq$ denotes inequality up to a universal constant independent of $n$.

The proof of the first statement follows trivially by the commutativity and associativity of the projective norm:

$$
\left(S_{1}\left(H_{A}\right) \widehat{\otimes} S_{1}\left(H_{B}\right)\right) \widehat{\otimes}\left(S_{1}\left(H_{A}\right) \widehat{\otimes} S_{1}\left(H_{B}\right)\right)=S_{1}\left(H_{A}^{\otimes 2}\right) \widehat{\otimes} S_{1}\left(H_{B}^{\otimes 2}\right) .
$$

We proceed similarly for the case of $k$ - tensors. To show the second part of Theorem 5.1, we must show that $\omega_{\text {qow }}\left(G^{\otimes k}\right)=\omega_{\text {qow }}(G)^{k}$. Since the inequality $\omega\left(G^{\otimes k}\right) \geq \omega(G)^{k}$ is trivial we have to focus on the other one. We recall that in this case the value of the game is given by

$$
\omega(G)^{1 / 2}=\|M\|_{S_{1}^{n} \otimes_{h} S_{1}^{n}}
$$

If the game $G$ corresponds to the linear map $\tilde{M}: M_{n} \rightarrow S_{1}^{n}$, then the game $G^{\otimes k}$ will correspond to the map $\tilde{M}^{\otimes k}: M_{n^{k}} \rightarrow S_{1}^{n^{k}}$. However, we have that

$$
M_{n^{k}} \simeq M_{n} \otimes_{\min } \cdots \otimes_{\min } M_{n} \quad \text { and } \quad S_{1}^{n^{k}} \simeq S_{1}^{n} \widehat{\otimes} \cdots \widehat{\otimes} S_{1}^{n} .
$$

We also recall that the Haagerup tensor product norm of $M \in S_{1}^{n} \otimes_{h} S_{1}^{n}$ coincides with the completely bounded factorization norm of the corresponding map $\tilde{M}: M_{n} \rightarrow S_{1}^{n}$ through $R$, the row Hilbert space. By Proposition 9.3.5 in [9] and the associativity of these tensor norms, we have the completely isometric identifications

$$
R=R \otimes_{\min } \cdots \otimes_{\min } R=R \otimes_{h} \cdots \otimes_{h} R=R \widehat{\otimes} \cdots \widehat{\otimes} R .
$$

Thus, given a completely bounded factorization $\tilde{M}=u v$

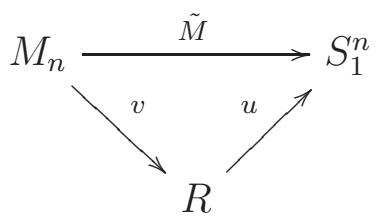

we obtain a completely bounded factorization $\tilde{M}^{\otimes k}=u^{\otimes k} v^{\otimes k}$ :

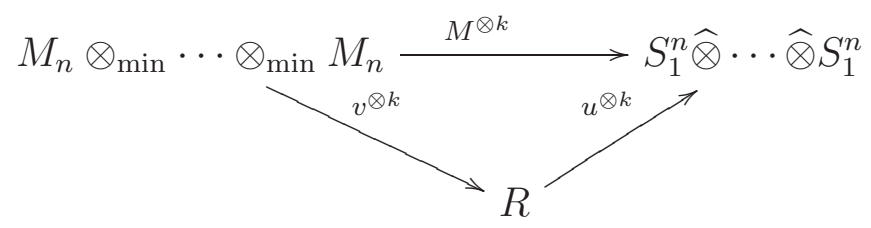

which provides the required norm estimate.

We were informed by Oded Regev and Thomas Vidick that one-way games can be seen as a special case of 2-round single-prover interactive proofs. With this reduction 
at hand, one could also deduce a perfect parallel repetition theorem from [12, Theorem 4.9]. Furthermore, once we have stated the connection between the one-way model of rank-one quantum games and the completely bounded norm (via Haagerup tensor norm) one could also study the multiplicativity properties via [26]. Still, we believe our proofs are potentially more illuminating and that the connection to the Haagerup tensor norm might have further implications.

To see that we do not have a perfect parallel repetition theorem for the entangled value of games we will construct a rank-one quantum game $G \in S_{1}^{n} \otimes S_{1}^{n}$ such that $\frac{\omega^{*}\left(G^{2}\right)}{\omega^{*}(G)^{2}} \succeq n^{2}$. As in Subsection 3.2 we prefer working directly with elements in $S_{1}^{n} \otimes S_{1}^{n}$. However, since the element we will give is very simple and completely explicit, one could easily write the associated rank-one quantum game.

Let's start by considering the element

$$
G=G_{C}+G_{R}=\sum_{i=1}^{n}\left(e_{i 1} \otimes e_{1 i}+e_{1 i} \otimes e_{i 1}\right) \in S_{1}^{n} \otimes S_{1}^{n},
$$

where $G_{R}$ and $G_{C}$ are the elements used in Subsection 3.2.

We will show that

$$
\|G\|_{S_{1}^{n} \otimes_{\min } S_{1}^{n}} \simeq 1,\|G\|_{S_{1}^{n} \widehat{\otimes} S_{1}^{n}} \simeq n \text { and }\|G \otimes G\|_{S_{1}^{n} \otimes_{\min } S_{1}^{n}} \simeq n,
$$

where here $\simeq$ denotes equality up to universal constants independent of $n$. Thus, having in mind Theorem 3.2, we will redefine

$$
\tilde{G}=\frac{G}{\|G\|_{S_{1}^{n} \widehat{\otimes} S_{1}^{n}}}
$$

to obtain a rank-one quantum game such that

$$
V(\tilde{G})=1, \omega^{*}(\tilde{G}) \simeq \frac{1}{n^{2}} \text { and } \omega^{*}(\tilde{G} \otimes \tilde{G}) \simeq \frac{1}{n^{2}}
$$

In particular, this game verifies

$$
\omega^{*}(\tilde{G} \otimes \tilde{G}) \geq n^{2} \omega^{*}(\tilde{G})^{2} .
$$

Since it is obvious that $1 \leq\|G\|_{S_{1}^{n} \otimes_{\min } S_{1}^{n}}$, the first estimate in Equation (5.2) follows trivially from the triangle inequality:

$$
\begin{aligned}
\|G\|_{S_{1}^{n} \otimes_{\min } S_{1}^{n}} & \leq\left\|G_{C}\right\|_{S_{1}^{n} \otimes_{\min } S_{1}^{n}}+\left\|G_{R}\right\|_{S_{1}^{n} \otimes_{\min } S_{1}^{n}} \\
& =\left\|\sum_{i=1} e_{i} \otimes e_{i}\right\|_{R_{n} \otimes_{\min } C_{n}}+\left\|\sum_{i=1} e_{i} \otimes e_{i}\right\|_{C_{n} \otimes_{\min } R_{n}}=2 .
\end{aligned}
$$

For the upper bound in the second estimate, we again use the triangle inequality:

$$
\begin{aligned}
\|G\|_{S_{1}^{n} \widehat{\otimes} S_{1}^{n}} & \leq\left\|G_{C}\right\|_{S_{1}^{n} \widehat{\otimes} S_{1}^{n}}+\left\|G_{R}\right\|_{S_{1}^{n} \widehat{\otimes} S_{1}^{n}} \\
& =\left\|\sum_{i=1} e_{i} \otimes e_{i}\right\|_{R_{n} \widehat{\otimes} C_{n}}+\left\|\sum_{i=1} e_{i} \otimes e_{i}\right\|_{C_{n} \widehat{\otimes} R_{n}}
\end{aligned}
$$




$$
=2\left\|\sum_{i=1} e_{i i}\right\|_{S_{1}^{n}}=2 n .
$$

Again, the inequality $n \leq\|G\|_{S_{1}^{n} \widehat{\otimes} S_{1}^{n}}$ is very easy.

Finally, we need to show the estimate on the tensor product

$$
G \otimes G=\left(G_{R} \otimes G_{R}\right)+\left(G_{R} \otimes G_{C}\right)+\left(G_{C} \otimes G_{R}\right)+\left(G_{C} \otimes G_{C}\right) .
$$

To do this, it is very useful to understand $G \otimes G$ as a map from $M_{n} \otimes_{\min } M_{n}$ to $S_{1}^{n} \widehat{\otimes} S_{1}^{n}$. Then, we must show that

$$
\left\|G \otimes G: M_{n} \otimes_{\min } M_{n} \rightarrow S_{1}^{n} \widehat{\otimes} S_{1}^{n}\right\|_{c b} \succeq n .
$$

Let's consider the element $x=\sum_{i=1}^{n} e_{1 i} \otimes e_{i 1} \in M_{n} \otimes M_{n}$. It is obvious that

$$
\|x\|_{M_{n} \otimes_{\min } M_{n}}=\left\|\sum_{i=1}^{n} e_{i} \otimes e_{i}\right\|_{R_{n} \otimes_{\min } C_{n}}=1 .
$$

On the other hand, trivial computations show that

$\left(G_{R} \otimes G_{R}\right)(x)=\left(G_{C} \otimes G_{R}\right)(x)=G_{C} \otimes G_{C}(x)=e_{11} \otimes e_{11} \quad$ and $G_{R} \otimes G_{C}(x)=\sum_{i=1}^{n} e_{i 1} \otimes e_{1 i}$.

Since

$$
\left\|\sum_{i=1}^{n} e_{i 1} \otimes e_{1 i}\right\|_{S_{1}^{n} \widehat{\otimes} S_{1}^{n}}=\left\|\sum_{i=1}^{n} e_{i} \otimes e_{i}\right\|_{R_{n} \widehat{\otimes} C_{n}}=n
$$

we conclude that $\|(G \otimes G)(x)\|_{S_{1}^{n} \widehat{\otimes} S_{1}^{n}} \succeq n$. Therefore,

$$
\left\|G \otimes G: M_{n} \otimes_{\min } M_{n} \rightarrow S_{1}^{n} \widehat{\otimes} S_{1}^{n}\right\|_{c b} \geq\left\|G \otimes G: M_{n} \otimes_{\min } M_{n} \rightarrow S_{1}^{n} \widehat{\otimes} S_{1}^{n}\right\| \succeq n .
$$

Using the triangle inequality one can show that $\left\|G \otimes G: M_{n} \otimes_{\min } M_{n} \rightarrow S_{1}^{n} \widehat{\otimes} S_{1}^{n}\right\|_{c b} \leq 4 n$. Indeed, one just has to show that each of the four operators of rank $n$ in (5.3) has completely bounded norm lower than or equal to $n$.

5.1. $O H_{n}$ - Games. The previous counterexample motivates the study of those games for which perfect parallel repetition is true or, at least, for which the violation in (5.1) cannot be large. We now present a large family of games $G \in S_{1}^{N} \otimes S_{1}^{N}$ for which

$$
\frac{\omega^{*}\left(G^{k}\right)}{\omega^{*}(G)^{k}} \leq C^{k}(1+\ln n)^{2 k}
$$

where $n$ is the rank of $G$ as an operator from $M_{N}$ to $S_{1}^{N}, k$ is any natural number and $C$ is a universal constant independent of $n$ and $k$. Our family will be formed by those maps $G: M_{N} \rightarrow S_{1}^{N}$ of rank $n$ verifying $\operatorname{tr}\left(G(x) x^{*}\right) \geq 0$ for every $x \in M_{n}$ (that is, $G$ is semidefinite positive). It is shown in [31] that in this case $G=\overline{V^{*}} V$ for certain $V: M_{N} \rightarrow O H_{n}$ such that $\|G\|_{c b}=\|V\|_{c b}^{2}$. This motivates our naming these games 
$O H_{n}$-games. According to Theorem 3.2 , it suffices to show that

$$
\frac{\left\|\otimes^{k} G: M_{N^{k}} \rightarrow S_{1}^{N^{k}}\right\|_{c b}}{\left\|G: M_{N} \rightarrow S_{1}^{N}\right\|_{c b}^{k}} \leq C^{\frac{k}{2}}(1+\ln n)^{k} .
$$

Our observation is that we have

$$
\left\|\otimes^{k} V: M_{N^{k}} \rightarrow O H_{n^{k}}\right\|_{c b} \leq \pi_{2}^{o}\left(\otimes^{k} V: M_{N^{k}} \rightarrow O H_{n^{k}}\right)=\pi_{2}^{o}\left(V: M_{N} \rightarrow O H_{n}\right)^{k} .
$$

Here, $\pi_{2}^{o}$ denotes the completely 2-summing norm (see [30], [17]). Therefore,

$$
\left\|\otimes^{k} G: M_{N^{k}} \rightarrow S_{1}^{N^{k}}\right\|_{c b} \leq \pi_{2}^{o}\left(V: M_{N} \rightarrow O H_{n}\right)^{2 k} .
$$

The main point is that $\pi_{2}^{o}(V) \leq c_{0} \sqrt{1+\ln n}\|V\|_{c b}$ for every map $V: M_{N} \rightarrow O H_{n}$, where $c_{0}$ is a universal constant independent of $n$ (see [17, Equation (2.3)]) and, hence, we obtain

$$
\left\|\otimes^{k} G: M_{N^{k}} \rightarrow S_{1}^{N^{k}}\right\|_{c b} \leq c_{0}^{2 k}(1+\ln n)^{k}\left\|G: M_{N} \rightarrow S_{1}^{N}\right\|_{c b}^{k}
$$

as we wanted.

Actually, the next construction shows that the order $(1+\ln n)^{2 k}$ in (5.4) is essentially optimal for these games. That is, for every $n$ we can construct a $O H_{n}$-game $G$ such that

$$
\frac{\omega^{*}\left(G^{k}\right)}{\omega^{*}(G)^{k}} \geq C_{1} C_{2}^{k} \frac{(1+\ln n)^{2 k}}{(1+k \ln n)^{2}}
$$

for every natural number $k$, where $C_{1}$ and $C_{2}$ are universal constants independent of $n$ and $k$. Therefore, even when Equation (5.4) tells us that perfect parallel repetition theorem is not far from being true for $\mathrm{OH}_{n^{-}}$games, it still fails for these kinds of games.

The construction relies on the following result proved in [17]:

Theorem 5.2. There exist universal constants $C_{0}>0$ and $C_{0}^{\prime}>0$ such that

1. For every $n$ we can find a natural number $N$, a complete contraction $u_{n}: O H_{n} \rightarrow$ $M_{N}$ and a linear map $w_{n}: M_{N} \rightarrow O H_{n}$ such that $\left\|w_{n}\right\|_{c b} \leq C_{0} \sqrt{\frac{n}{1+\ln n}}$ and verifying $w_{n} \circ u_{n}=i d: \ell_{2}^{n} \rightarrow \ell_{2}^{n}$.

2. The previous factorization is optimal. That is, for every $n$ and for every maps $u: O H_{n} \rightarrow B\left(\ell_{2}\right), w: B\left(\ell_{2}\right) \rightarrow O H_{n}$ verifying $w \circ u=i d: \ell_{2}^{n} \rightarrow \ell_{2}^{n}$ we have $\|u\|_{c b}\|w\|_{c b} \geq C_{0}^{\prime} \sqrt{\frac{n}{1+\ln n}}$.

The above result is Corollary 4.11 in [17] (see also [21]). The inequality missing in the statement of that result follows immediately from combining the argument of Corollary 4.11 (i.e., $\left.\gamma_{\infty}\left(i d_{O H_{n}}\right) \pi_{1}^{o}\left(i d_{O H_{n}}\right)=n\right)$ with the estimates for $\pi_{1}^{o}\left(i d_{O H_{n}}\right)$ given by Corollary 4.8 and Proposition 4.9.

We want to show that

$$
\frac{\left\|\otimes^{k} G: M_{N^{k}} \rightarrow S_{1}^{N^{k}}\right\|_{c b}}{\left\|G: M_{N} \rightarrow S_{1}^{N}\right\|_{c b}^{k}} \geq \sqrt{C_{1}} C_{2}^{\frac{k}{2}} \frac{(1+\ln n)^{k}}{1+k \ln n} .
$$


Fixing $n$ and $k$ we consider the map $w_{n}: M_{N} \rightarrow O H_{n}$ of Theorem 5.2. The first property above tells us that

$$
\left\|w_{n}: M_{N} \rightarrow O H_{n}\right\|_{c b}^{k} \leq C_{0}^{k}\left(\sqrt{\frac{n}{1+\ln n}}\right)^{k} .
$$

On the other hand, since $\left\|\otimes^{k} u_{n}: O H_{n^{k}} \rightarrow M_{N^{k}}\right\|_{c b} \leq 1$, the second part of Theorem 5.2 applied to $O H_{n^{k}}$ tells us that

$$
\left\|\otimes^{k} w_{n}: M_{N^{k}} \rightarrow O H_{n^{k}}\right\| \geq C_{0}^{\prime} \sqrt{\frac{n^{k}}{1+k \ln n}} .
$$

Let's consider now the $O H_{n^{-}}$game defined by the map

$$
G: \overline{w_{n}^{*}} \circ w_{n}: M_{N} \rightarrow \overline{O H_{n}} \simeq O H_{n}^{*} \rightarrow S_{1}^{N} \text {. }
$$

Note that

$$
\otimes^{k} G=\left(\otimes^{k} \overline{w_{n}^{*}}\right) \circ\left(\otimes^{k} w_{n}\right): M_{N^{k}} \rightarrow \overline{O H_{n^{k}}} \simeq O H_{n^{k}}^{*} \rightarrow S_{1}^{N^{k}} .
$$

Then, as we mentioned before,

$$
\left\|G: M_{N} \rightarrow S_{1}^{N}\right\|_{c b}=\left\|w_{n}: M_{N} \rightarrow O H_{n}\right\|_{c b}^{2}
$$

and

$$
\left\|\otimes^{k} G: M_{N^{k}} \rightarrow S_{1}^{N^{k}}\right\|_{c b}=\left\|\otimes^{k} w_{n}: M_{N^{k}} \rightarrow O H_{n^{k}}\right\|_{c b}^{2} .
$$

Therefore,

$$
\frac{\left\|\otimes^{k} G: M_{N^{k}} \rightarrow S_{1}^{N^{k}}\right\|_{c b}}{\left\|G: M_{N} \rightarrow S_{1}^{N}\right\|_{c b}^{k}} \geq \frac{\left(C_{0}^{\prime}\right)^{2} \frac{n^{k}}{1+k \ln n}}{C_{0}^{2 k}\left(\frac{n}{1+\ln n}\right)^{k}}=\left(C_{0}^{\prime}\right)^{2}\left(\frac{1}{C_{0}}\right)^{2 k} \frac{(1+\ln n)^{k}}{1+k \ln n} .
$$

Remark 5.1. The preceding construction can be altered to provide also the maximal value of the game involved. According to [17] (see also [13]), for every $n$ we can find an $N$ and a complete embedding $j_{n}: O H_{n} \hookrightarrow S_{1}^{N}$ such that $\left\|j_{n}\right\|\left\|j_{n}^{-1}\right\| \leq C$ for certain universal constant $C$ different from the ones appearing above. On the other hand, it was proved in [17] (see also [21]) that

$$
\pi_{1}^{o}\left(i d: O H_{n} \rightarrow O H_{n}\right) \simeq \sqrt{n(1+\ln n)},
$$

where $\simeq$ denotes equality up to universal constants and $\pi_{1}^{o}$ denotes the completely 1 summing norm (see [30]). Thus, defining the map

$$
G=j_{n} \circ j_{n}^{*}: M_{N} \rightarrow S_{1}^{N}
$$

it can be deduced that the associated tensor $G \in S_{1}^{N} \otimes S_{1}^{N}$ verifies

$$
\|G\|_{S_{1}^{N} \otimes_{\min } S_{1}^{N}} \simeq 1 \text { and }\|G\|_{S_{1}^{N} \widehat{\otimes} S_{1}^{N}} \simeq \sqrt{n(1+\ln n)} .
$$

Now, since the projection constant of $O H_{n}$ is of order $\sqrt{\frac{n}{1+\ln n}}$ (see [17]), we can find a map $P_{n}: S_{1}^{N} \rightarrow O H_{n}$ such that $\|P\|_{c b} \preceq \sqrt{\frac{n}{1+\ln n}}$ and $P_{\circ} j_{n}=i d_{O H_{n}}$. Then, following exactly the same argument as above one can show that the map $G$ also verifies Equation 
(5.6) (with different constants). If we normalize, we obtain a rank-one quantum game

$\tilde{G}:=\frac{G}{\sqrt{n(1+\ln n)}}$ with maximal value $V(\tilde{G}) \simeq 1$, entangled value $\omega^{*}(\tilde{G}) \simeq \frac{1}{n(1+\ln n)}$ and verifying Equation (5.6).

\section{Discussion AND OPEN QUESTIONS}

In this work we showed that operator spaces are ideally suited to study one rankone quantum games. Specifically, we show that the entangled value and the entangled value with one way communication of a rank-one quantum game can be exactly described by the minimal and the Haagerup operator space tensor norms respectively. With this connection at hand, we used some deep results on operator spaces to study two main problems in the area: computability and approximability of these values and its behavior with respect to the parallel repetition of the game. Considering the entangled value with one way communication we proved that this value can be efficiently computed and that it verifies a perfect parallel repetition when we are dealing with rank-one quantum games. Therefore, both problems are completely solved in an optimal way. Regarding the entangled value of a rank-one quantum game, on the one hand we showed that it can be efficiently approximated up to a multiplicative factor of 4 . Therefore, it would be interesting to know if such a value can be actually efficiently computed or, on the contrary, if there is any kind of NP hardness result about it. On the other hand, we also proved that the entangled value does not verify a perfect parallel repetition theorem on rank-one quantum games. One should then study whether a (strong) parallel repetition holds for these kinds of games.

Finally, as we mentioned before the techniques used in this paper also apply to the general case of quantum games. In particular, one can describe general quantum games via certain tensor norms in the framework of operator spaces. However, the statement of similar results for general quantum games requires an improvement of some results in the operator space setting.

\section{ACKNOWLEDGMENTS}

During the process of preparing this work we learned that Oded Regev and Thomas Vidick came up with similar connections while considering different kinds of games 3 . We must thank both authors for a very generous and complete reading of our preliminary draft.

\section{REFERENCES}

[1] S. Arora, C. Lund, R. Motwani, M. Sudan and M. Szegedy, Proof verification and the hardness of approximation problems. J. ACM, 45(3):501-555 (1998).

[2] S. Arora and S. Safra, Probabilistic checking of proofs: a new characterization of NP, J. ACM, 45(1):70-122 (1998).

\footnotetext{
${ }^{3}$ Work in progress.
} 
[3] M. Ben-Or, A. Hassidim and H. Pilpel, Quantum Multiprover Interactive Proofs with Communicating Provers, In Proc. 49th IEEE Symp. on Foundations of Computer Science (FOCS), pages 467-476 (2008).

[4] H. Buhrman, N. Chandran, S. Fehr, R. Gelles, V. Goyal, R. Ostrovsky and C. Schaffner, PositionBased Quantum Cryptography: Impossibility and Constructions, In Advances in Cryptology CRYPTO 2011. Lecture Notes in Computer Science. Springer-Verlag, (2011).

[5] J.T. Chan, Facial Structure of the Trace Class, Arch. Math. 64 (1994), no. 3, 185-187.

[6] R. Cleve, W. Slofstra, F. Unger and S. Upadhyay, Strong parallel repetition theorem for quantum XOR proof systems. In Proceedings of 22nd IEEE Conference on Computational Complexity, pages 282-299, (2007). quant-ph/0608146.

[7] I. Devetak, M. Junge, C. King and M. B. Ruskai, Multiplicativity of completely bounded p-norms implies a new additivity result, Comm. Math. Phys. 266, no. 1, 37-63 (2006).

[8] E. Effros, M. Junge and Z-J. Ruan, Integral mappings and the principle of local reflexivity for noncommutative $L_{1}$-spaces, Ann. of Math. 151, 59-92 (2000).

[9] E. Effros and Z.-J. Ruan, Operator Spaces, London Mathematical Society Monographs New Series, Vol. 23, Oxford University Press, Oxford, 2000.

[10] U. Feige, On the success probability of two provers in one-round proof systems, In Proc. 6th IEEE Structure in Complexity Theory, pages 116-123 (1991).

[11] M. Grotschel, L. Lovasz and A. Schrijver, Geometric algorithms and combinatorial optimization, volume 2 of Algorithms and Combinatorics Springer-Verlag, Berlin, second edition, (1993).

[12] G. Gutoski, Quantum Strategies and Local Operations, arXiv:1003.0038 (2010).

[13] U. Haagerup and M. Musat, On the best constants in noncommutative Khintchine-type inequalities, J. Funct. Anal. 250 (2007), no. 2, 588-624.

[14] U. Haagerup and M. Musat, The Effros-Ruan conjecture for bilinear forms on $C^{*}$-algebras, Invent. Math. 174 (2008), 139-163.

[15] N. Johnston, D. W. Kribs and V. I. Paulsen, Computing Stabilized Norms for Quantum Operations via the Theory of Completely Bounded Maps, Quantum Information and Computation, 9 16-35. (2009).

[16] N. Johnston, D.W. Kribs, V. I. Paulsena and R. Pereira, Minimal and Maximal Operator Spaces and Operator Systems in Entanglement Theory, J. Funct. Anal. 260, 2407-2423 (2011).

[17] M. Junge, Embedding of the operator space $O H$ and the logarithmic "little Grothendieck inequality", Invent. Math. 161 (2005), no. 2, 225-286.

[18] M. Junge, M. Navascues, C. Palazuelos, D. Pérez-García, V. B. Scholz and R. F. Werner, Connes' embedding problem and Tsirelson's problem, J. Math. Phys 52, 012102 (2011).

[19] M. Junge and C. Palazuelos, Large violation of Bell inequalities with low entanglement, Comm. Math. Phys. 306 (3), 695-746 (2011).

[20] M. Junge, C. Palazuelos, D. Pérez-García, I. Villanueva and M.M. Wolf, Operator Space theory: a natural framework for Bell inequalities, Phys. Rev. Lett. 104, 170405 (2010).

[21] M. Junge and Q. Xu, Representation of certain homogeneous Hilbertian operator spaces and applications, Invent. Math. 179, no. 1, 75-118 (2010).

[22] J. Kempe, H. Kobayashi, K. Matsumoto, B. Toner and T. Vidick, Entangled Games are Hard to approximate, Proc. 49th FOCS'08, p. 447-456 (2008)

[23] J. Kempe and O. Regev, No Strong Parallel Repetition with Entangled and Non-signaling Provers, In Proc. 25th IEEE Conference on Computational Complexity (CCC), pages 7-15 (2010). 
[24] J. Kempe, O. Regev and B. Toner, Unique Games with Entangled Provers are Easy, In Proc. 49th IEEE Symp. on Foundations of Computer Science (FOCS), pages 457-466 (2008).

[25] J. Kempe and T. Vidick, Parallel Repetition of Entangled Games, To appear in STOC'11.

[26] A. Kitaev and J. Watrous, Parallelization, amplification, and exponential time simulation of quantum interactive proof systems, In Proceedings of the Thirty-second Annual ACM Symposium on the Theory of Computing, Portland OR, 608-617 (2000).

[27] H. Kobayashi and K. Matsumoto, Quantum multi-prover interactive proof systems with limited prior entanglement. Journal of Computer and System Sciences 66(3), 429-450 (2003).

[28] D. Leung, B. Toner and J. Watrous. Coherent state exchange in multi-prover quantum interactive proof systems. arXiv:0804.4118 quant-ph].

[29] D. Pérez-García, M. M. Wolf, C. Palazuelos, I. Villanueva and M. Junge, Unbounded violation of tripartite Bell inequalities, Comm. Math. Phys. 279 (2), 455-486 (2008).

[30] G. Pisier, Non-Commutative Vector Valued $L_{p}$-Spaces and Completely -Summing Maps, Asterisque, 247 (1998).

[31] G. Pisier, Introduction to operator space theory. London Mathematical Society Lecture Note Series, 294. Cambridge University Press, Cambridge, 2003.

[32] G. Pisier, Grothendieck's theorem, past and present. To appear in Bull. Amer. Math. Soc. (N.S.), preprint available at http://arxiv.org/abs/1101.4195 (2011).

[33] G. Pisier and D. Shlyakhtenko, Grothendieck's theorem for operator spaces, Invent. Math. 150 (2002), no. 1, 185-217.

[34] A. Rapaport and Ta-Shma, On the power of quantum, one round, two prover interactive proof systems, Quantum Inf. Process. 6 (2007), no. 6, $445 Đ 459$.

[35] R. Raz, A parallel repetition theorem, SIAM Journal on Computing, 27:763-803 (1998).

[36] J. Watrous, Semidefinite programs for completely bounded norms, Theory of computing, 5, 217-238 (2009).

[37] John Watrous, PSPACE has constant-round quantum interactive proof systems, Theoretical Computer Science 292(3), 575-588 (2003).

\section{SOME PROOFS}

\subsection{Proof of Lemma 3.3.}

Proof of Lemma 3.3. For the first part of the proof we will use that, given $M \in S_{1}\left(H_{A}\right) \otimes$ $S_{1}\left(H_{B}\right)$

$$
\|M\|_{\min }=\sup \|(T \otimes S)(M)\|_{B\left(H_{A^{\prime}}\right) \otimes_{\min } B\left(H_{B^{\prime}}\right)},
$$

where the supremum is taken over all finite dimensional Hilbert spaces $H_{A^{\prime}}$ and $H_{B^{\prime}}$; and all complete contractions $\hat{T}: S_{1}\left(H_{A}\right) \rightarrow B\left(H_{A^{\prime}}\right)$ and $\hat{S}: S_{1}\left(H_{B}\right) \rightarrow B\left(H_{B^{\prime}}\right)$. This can be easily deduced from the definition of the min norm. Now, given such a $\hat{T}$, the associated tensor $T$ can be seen as an element in the unit ball of $B\left(H_{A}\right) \otimes_{\min } B\left(H_{A^{\prime}}\right)=B\left(H_{A} \otimes H_{A^{\prime}}\right)$ (recall that we are in finite dimension). Since we are interested in the extremal points we can restrict to unitaries $U_{A A^{\prime}}$ in $B\left(H_{A} \otimes H_{A^{\prime}}\right)$. Using the same reasoning for $S$ we can 
restrict to unitaries $V_{B B^{\prime}}$ in $B\left(H_{B} \otimes H_{B^{\prime}}\right)$. Let

$$
U=\sum_{i} u_{i}^{1} \otimes u_{i}^{2} \text { and } V=\sum_{j} v_{j}^{1} \otimes v_{j}^{2}
$$

The corresponding complete contractions $\hat{U}: S_{1}\left(H_{A}\right) \rightarrow B\left(H_{A^{\prime}}\right)$ and $\hat{V}: S_{1}\left(H_{B}\right) \rightarrow$ $B\left(H_{B^{\prime}}\right)$ are given by (using the scalar pairing)

$$
\hat{U}(x)=\sum_{i} \operatorname{tr}\left(\left(u_{i}^{1}\right)^{t r} x\right) u_{i}^{2} \text { and } \hat{V}(y)=\sum_{j} \operatorname{tr}\left(\left(v_{j}^{1}\right)^{\operatorname{tr}} y\right) v_{j}^{2} .
$$

We thus need to compute

$$
\sup _{U, V}\|(\hat{U} \otimes \hat{V})(M)\|_{B\left(H_{A^{\prime}} \otimes H_{B^{\prime}}\right)}
$$

where the supremum is over unitaries $U_{A A^{\prime}} \in B\left(H_{A} \otimes H_{A^{\prime}}\right), V_{B B^{\prime}} \in B\left(H_{B} \otimes H_{B^{\prime}}\right)$. We write $M=\sum_{k} x_{k} \otimes y_{k}$. If $|\xi\rangle=\sum_{i, j} \xi_{i j}|i\rangle|j\rangle$, where $\{|i\rangle|j\rangle\}_{i, j}$ is an orthonormal basis for $H_{A^{\prime}} \otimes H_{B^{\prime}}$, then we write $|\bar{\xi}\rangle$ for $\sum_{i, j} \overline{\xi_{i j}}|i\rangle|j\rangle$. The above operator norm can then be evaluated by taking the supremum over $|\xi\rangle$ and $|\eta\rangle$ in the unit ball of $H_{A^{\prime}} \otimes H_{B^{\prime}}$ to get

$$
\begin{array}{r}
\sup _{|\xi\rangle,|\eta\rangle}\left|\sum_{i, j, k} \operatorname{tr}\left(\left(u_{i}^{1}\right)^{\operatorname{tr}} x_{k}\right) \operatorname{tr}\left(\left(v_{j}^{1}\right)^{\operatorname{tr}} y_{k}\right)\left\langle\bar{\eta}\left|u_{i}^{2} \otimes v_{j}^{2}\right| \bar{\xi}\right\rangle\right| \\
=\sup _{|\xi\rangle,|\eta\rangle}\left|\sum_{i, j, k} \operatorname{tr}\left(\left(u_{i}^{1}\right)^{\operatorname{tr}} x_{k}\right) \operatorname{tr}\left(\left(v_{j}^{1}\right)^{\operatorname{tr}} y_{k}\right)\left\langle\xi\left|\left(u_{i}^{2}\right)^{\operatorname{tr}} \otimes\left(v_{j}^{2}\right)^{\operatorname{tr}}\right| \eta\right\rangle\right| .
\end{array}
$$

As $U^{t r}$ and $V^{t r}$ are also unitaries, we have that

$$
\|M\|_{S_{1}\left(H_{A}\right) \otimes_{\min } S_{1}\left(H_{B}\right)}=\sup \left\{\left|\operatorname{tr}_{A B}\left(\left\langle\xi\left|U_{A A^{\prime}} \otimes V_{B B^{\prime}}\right| \eta\right\rangle M\right)\right|\right\},
$$

where the supremum is taken over $|\xi\rangle,|\eta\rangle$ in the unit ball of $H_{A^{\prime}} \otimes H_{B^{\prime}}$, unitaries $U \in$ $B\left(H_{A} \otimes H_{A^{\prime}}\right)$ and $V \in B\left(H_{B} \otimes H_{B^{\prime}}\right)$.

For the second case, we will use that given $M \in S_{1}\left(H_{A}\right) \otimes S_{1}\left(H_{B}\right)$,

$$
\|M\|_{h}=\sup \|(T \odot S)(M)\|_{B\left(H_{A^{\prime}}\right)}
$$

where the sup is taken over all complete contractions $T: S_{1}\left(H_{A}\right) \rightarrow B\left(H_{A^{\prime}}\right)$ and $S$ : $S_{1}\left(H_{B}\right) \rightarrow B\left(H_{A^{\prime}}\right)$ and $(T \odot S)(x \otimes y)=T(x) S(y)$ (see [31, Chapter 5]). In the same way as before, we can assume that $T$ and $S$ are operators associated to unitaries $U_{A A^{\prime}}$ in $B\left(H_{A} \otimes H_{A^{\prime}}\right)$ and $V_{B A^{\prime}}$ in $B\left(H_{B} \otimes H_{A^{\prime}}\right)$ respectively. Proceeding as above, we see that

$$
\|(T \odot S)(M)\|_{B\left(H_{A^{\prime}}\right)}=\sup \left\{\left|\operatorname{tr}_{A B}\left(\left\langle\xi\left|V_{B A^{\prime}} U_{A A^{\prime}}\right| \eta\right\rangle M\right)\right|\right\},
$$

where the supremum is taken over $|\xi\rangle,|\eta\rangle$ in the unit ball of $H_{A^{\prime}}$. As the transpose is an anti-homomorphism, there is a switch in the order of the product from $U V$ to $V U$. Thus we have that

$$
\|M\|_{S_{1}\left(H_{A}\right) \otimes_{h} S_{1}\left(H_{B}\right)}=\sup \left\{\left|\operatorname{tr}_{A B}\left(\left\langle\xi\left|V_{B A^{\prime}} U_{A A^{\prime}}\right| \eta\right\rangle M\right)\right|\right\}
$$


The final equality in the statement of the lemma follows as the unit ball of $B\left(H_{A} \otimes H_{B}\right)$ is the convex hull of the unitaries on $H_{A} \otimes H_{B}$. Suppose that $x$ is an element in $B\left(H_{A} \otimes H_{B}\right)_{1}$ with $x=\sum_{i=1}^{k} \lambda_{i} W_{i}$, where $W_{k}$ are unitaries on $H_{A} \otimes H_{B}, \lambda_{i} \geq 0, \sum_{i=1}^{k} \lambda_{i}=1$. We set $W=\sum_{i=1}^{k} W_{i} \otimes|i\rangle\langle i|$; we then have that $W$ is a unitary on $H_{A} \otimes H_{B} \otimes \mathbb{C}^{k}$. Setting $|\xi\rangle=|\eta\rangle=\sum_{i=1}^{k} \sqrt{\lambda_{i}}|i\rangle$, we have $x=\langle\xi|W| \eta\rangle$.

7.2. Proof of Lemma 3.4. We begin by fixing some notation.

$$
\begin{array}{rll}
|\psi\rangle=\sum_{i} \lambda_{i}|i\rangle\left|\alpha_{i}\right\rangle,\left|\alpha_{i}\right\rangle \in H_{A} \otimes H_{B}, \quad & \|\left|\alpha_{i}\right\rangle \|_{2}=1, \quad \lambda_{i} \geq 0, \quad & \sum_{i} \lambda_{i}^{2}=1 \\
|\gamma\rangle=\sum_{i} \mu_{i}|i\rangle\left|\beta_{i}\right\rangle,\left|\beta_{i}\right\rangle \in H_{A} \otimes H_{B}, & \|\left|\beta_{i}\right\rangle \|_{2}=1, \quad \mu_{i} \geq 0, & \sum_{i} \mu_{i}^{2}=1
\end{array}
$$

and write $M$ for the associated matrix $\operatorname{tr}_{C}(|\psi\rangle\langle\gamma|) \in B\left(H_{A} \otimes H_{B}\right)$. We know that $\|M\|_{S_{1}\left(H_{A} \otimes H_{B}\right)} \leq 1$ (see Proposition 3.1).

We will use the following result from [5]:

Theorem 7.1. A subset $F$ is a proper closed face of the the unit ball of $S_{1}^{n}$ if and only if there exists a nonzero partial isometry $U$ such that

$$
F=\left\{U P: P \geq 0,\|P\|_{1}=1 \text {, and } \operatorname{ker} P \supseteq \operatorname{ker} U\right\} .
$$

Proof of Lemma 3.4. Suppose that such a unitary $U$ exists and that $\lambda_{i}=\mu_{i}$ for all $i$. In this situation, we have that

$$
M=\sum_{i} \lambda_{i}^{2} U\left|\beta_{i}\right\rangle\left\langle\beta_{i}\right|,
$$

and then $\operatorname{tr}\left(U^{*} M\right)=\sum_{i} \lambda_{i}^{2}=1$. By the duality between $S_{1}^{n}$ and $S_{\infty}^{n}$ (and as we know that $\left.\|M\|_{\wedge} \leq 1\right)$, we have that $\|M\|_{\wedge}=1$.

For the converse, first note that we can assume that $\lambda_{i}$ and $\mu_{i}$ are non negative real numbers for every $i$. Let's assume that $\|M\|_{\wedge}=1$. Then

$$
1=\| \sum_{i} \lambda_{i} \mu_{i}\left|\alpha_{i}\right\rangle\left\langle\beta_{i}\right| \|_{1} \leq \sum_{i} \lambda_{i} \mu_{i} \leq\left(\sum_{i} \lambda_{i}^{2}\right)^{\frac{1}{2}}\left(\sum_{i} \mu_{i}^{2}\right)^{\frac{1}{2}} \leq 1
$$

Equality in the Cauchy-Schwartz inequality implies $\lambda_{i}=\mu_{i}$ for all $i$. We are thus considering the matrix $\sum_{i} \lambda_{i}^{2}\left|\alpha_{i}\right\rangle\left\langle\beta_{i}\right|$, a convex combination of elements in the unit sphere of $S_{1}^{n}$. If $\|M\|_{\wedge}=1$, we must have that $\left|\alpha_{i}\right\rangle\left\langle\beta_{i}\right|$ all lie in the same face of the unit ball of $S_{1}^{n}$. But in this case, there exist $P \geq 0, \operatorname{tr}(P)=1$, and a partial isometry $U$ such that

$$
\left|\alpha_{i}\right\rangle\left\langle\beta_{i}\right|=U P, \quad \text { for all } i \text {. }
$$

We then have that $P=U^{*}\left|\alpha_{i}\right\rangle\left\langle\beta_{i}\right|$. By positivity of $P$, we must have $\left|\alpha_{i}\right\rangle=U\left|\beta_{i}\right\rangle$. As $H_{A} \otimes H_{B}$ is finite dimensional, there exists a unitary $\tilde{U}$ such that $\left.U\right|_{\operatorname{supp} U}=\left.\tilde{U}\right|_{\operatorname{supp} U}$.

\subsection{Proof of Lemma 3.5.}


Proof of Lemma 3.5. Assume that the rank-one quantum game is a Schur game, i.e., after fixing suitable orthonormal bases, the element $\hat{M}_{A B}$ in $C B\left(M_{n}, S_{1}^{n}\right)$ corresponding to the game is of the form $\left(x_{i j}\right) \mapsto\left(t_{i j} x_{i j}\right)$. We now examine the relationship between the element $M_{A B} \in C B\left(M_{n}, S_{1}^{n}\right)$ and the states $|\psi\rangle$ and $|\gamma\rangle$.

By taking a partial trace over $H_{C}$, we obtain

$$
\operatorname{tr}_{C}|\psi\rangle\left\langle\gamma\left|=\sum_{r, s, t, u, v} \alpha_{r s t} \overline{\beta_{u v t}}\right| r\right\rangle\left\langle\left. u\right|_{A} \otimes \mid s\right\rangle\left\langle\left. v\right|_{B} .\right.
$$

Using the identification $S_{1}^{n} \otimes_{\min } S_{1}^{n} \simeq C B\left(M_{n}, S_{1}^{n}\right)$ and using the scalar pairing to identify $M_{n}^{*}$ and $S_{1}^{n}$, we apply this to $|i\rangle\langle j| \in M_{n}$ to get

$$
\begin{aligned}
\left(\operatorname{tr}_{C}|\psi\rangle\langle\varphi|\right)(|i\rangle\langle j|) & =\sum_{s, v}\left(\sum_{r, u, t} \alpha_{r s t} \overline{\beta_{u v t}}\langle i \mid r\rangle\langle u \mid j\rangle\right)|s\rangle\langle v| \\
& =\sum_{s, t, v} \alpha_{i s t} \overline{\beta_{j v t}}|s\rangle\langle v| .
\end{aligned}
$$

For this to be a Schur multiplier, the above sum must equal $t_{i j}|i\rangle\langle j|$. Replacing $\alpha_{i s t}$ by $\delta_{s t} \alpha_{i s t}$ and $\beta_{j v t}$ by $\delta_{j v} \beta_{j v t}$ will not alter $\operatorname{tr}_{C}|\psi\rangle\langle\gamma|$ and thus we can express the states as

$$
\begin{aligned}
|\psi\rangle & =\sum_{i, t} \alpha_{i i t}|i\rangle_{A}|i\rangle_{B}|t\rangle_{C}, \\
|\gamma\rangle & =\sum_{j, t} \beta_{j j t}|j\rangle_{A}|j\rangle_{B}|t\rangle_{C},
\end{aligned}
$$

with $\sum_{i, t}\left|\alpha_{i i t}\right|^{2} \leq 1, \sum_{j, t}\left|\beta_{j j t}\right|^{2} \leq 1$. The reverse implication is trivial.

Departamento de Análisis Matemático and imi, Universidad Complutense de Madrid, 28040, MADRID, SPAIN

E-mail address: tomcooney1@gmail.com

Department of Mathematics, University of Illinois, Urbana, IL 61801, USA

E-mail address: junge@math.uiuc.edu

Instituto de Ciencias Matemáticas, CSiC-UAM-UC3M-UCM, CSiC, 28049, Madrid. Spain

E-mail address: carlospalazuelos@icmat.es

Departamento de Análisis Matemático and imi, Universidad Complutense de Madrid, 28040, MAdrid, Spain

E-mail address: dperez@mat.ucm.es 